\title{
Asthma-associated genetic variants induce IL33 differential expression through a novel regulatory region.
}

Ivy Aneas

University of Chicago

Donna Decker

University of Chicago

Chanie Howard

University of Chicago

Debora Sobreira

University of Chicago

Noboru Sakabe

University of Chicago

Kelly Blaine

University of Chicago

Michelle Stein

University of Chicago

Cara Hrusch

University of Chicago

Lindsey Montefiori

University of Chicago

Juan Tena

Centro Andaluz de Biología del Desarrollo https://orcid.org/0000-0001-8165-7984

Kevin Magnaye

University of Chicago https://orcid.org/0000-0002-9425-1825

Selene Clay

University of Chicago https://orcid.org/0000-0002-7204-9570

James Gern

the University of Wisconsin-Madison

Daniel Jackson

Department of Pediatrics, University of Wisconsin, Madison, WI

Matthew Altman

University of Washington https://orcid.org/0000-0002-1784-8505

Edward Naurekas 
University of Chicago

Douglas Hogarth

University of Chicago

Steven White

University of Chicago

José Luis Gómez-Skarmeta

Centro Andaluz de Biología del Desarrollo (CABD)

Nathan Schoettler

University of Chicago https://orcid.org/0000-0001-9851-6352

Carole Ober

University of Chicago

Anne Sperling

University of Chicago

Marcelo Nobrega ( $\square$ mnobrega@bsd.uchicago.edu )

Department of Human Genetics, University of Chicago https://orcid.org/0000-0002-2134-9661

\section{Article}

Keywords:

Posted Date: October 9th, 2020

DOI: https://doi.org/10.21203/rs.3.rs-84333/v1

License: () (1) This work is licensed under a Creative Commons Attribution 4.0 International License. Read Full License

Version of Record: A version of this preprint was published at Nature Communications on October 21st, 2021. See the published version at https://doi.org/10.1038/s41467-021-26347-z. 


\section{Asthma-associated genetic variants induce IL33 differential expression through a novel regulatory region}

Ivy Aneas ${ }^{1 \star \wedge}$, Donna C. Decker ${ }^{2 \star}$, Chanie L. Howard ${ }^{3}$, Débora R. Sobreira ${ }^{1}$, Noboru J. Sakabe $^{1}$, Kelly M. Blaine ${ }^{2}$, Michelle M. Stein ${ }^{1}$, Cara L. Hrusch ${ }^{2}$, Lindsey E. Montefiori ${ }^{1}$, Juan Tena ${ }^{4}$, Kevin M. Magnaye ${ }^{1}$, Selene M. Clay ${ }^{1}$ James E. Gernn, Daniel J. Jackson ${ }^{5}$, Matthew C. Altman ${ }^{6}$, Edward T. Naureckas ${ }^{2}$, Douglas K. Hogarth², Steven R. White ${ }^{2}$, 8 Jose Luis Gomez-Skarmeta ${ }^{4}$, Nathan Schoetler ${ }^{2}$, Carole Ober ${ }^{1}$, Anne I. Sperling ${ }^{2,3 * \wedge,}$ 9 Marcelo A. Nobrega ${ }^{1 * \wedge}$,

11 Author affiliations:

12 1- Department of Human Genetics, University of Chicago, Chicago, IL 60637, USA.

13 2- Department of Medicine, Section of Pulmonary and Critical Care Medicine, University of 14 Chicago, Chicago, IL 60637, USA.

15 3- Committee on Immunology, University of Chicago, Chicago IL 60637

16 4- Centro Andaluz de Biología del Desarrollo (CSIC/UPO/JA), Universidad Pablo de Olavide, 17 Seville 41013, Spain.

18 5- Department of Pediatrics, University of Wisconsin School of Medicine and Public Health,

19 Madison, WI 53726, USA

20 6- Division of Allergy and Infectious Diseases, Department of Medicine, University of

21 Washington, Seattle, WA 98195, USA

22 *These authors contributed equally to this study

^ Corresponding Authors:

Anne I. Sperling

Section of Pulmonary and Critical Care, Dept of Medicine

University of Chicago

924 E. 57th Street

Chicago, IL 60637

asperlin@uchicago.edu

Marcelo A. Nobrega

Department of Human Genetics

University of Chicago

920 E 58 ${ }^{\text {th }}$ St CLSC 515-E

nobrega@uchicago.edu

Ivy Aneas

Department of Human Genetics

University of Chicago

920 E 58 ${ }^{\text {th }}$ St CLSC 515-E

ianeas@bsd.uchicago.edu 


\section{ABSTRACT}

44 Genome-wide association studies (GWAS) have implicated the IL33 locus in asthma,

45 but the underlying mechanisms remain unclear. Here, we identify a $5 \mathrm{~kb}$ region within

46 the GWAS-defined segment that acts as a strong regulatory element in vivo and in vitro.

47 Chromatin conformation capture showed that this $5 \mathrm{~kb}$ region loops to the IL33

48 promoter, potentially regulating its expression. We show that genotype at the asthma-

49 associated SNP rs1888909, located within the $5 \mathrm{~kb}$ region, is associated with IL33 gene

50 expression in human airway epithelial cells and IL-33 protein expression in human

51 plasma, potentially through differential binding of OCT-1 (POU2F1) to the asthma-risk

52 allele. Our data demonstrate that asthma-associated variants at the IL33 locus mediate

53 allele-specific regulatory activity and IL33 expression, providing a novel mechanism

54 through which a regulatory SNP contributes to genetic risk of asthma. 
Introduction

Asthma is a common and chronic inflammatory disease of the airways, with significant contributions from both genetic and environmental factors. Genetic factors

59 account for more than half of the overall disease liability ${ }^{1}$ and GWAS have discovered

60 more than 60 loci contributing to asthma disease risk ${ }^{2}$, with most of the associated

61 variants located in noncoding regions. Linking these noncoding variants to genes and

62 understanding the mechanisms through which they impart disease risk remains an

63 outstanding task for nearly all asthma GWAS loci.

64 Among the most highly replicated asthma loci are variants near the genes

65 encoding the cytokine IL-33 on chromosome 9p24.1 and its receptor, ST2 (encoded by

$66 I L 1 R L 1$ ), on chromosome $2 q 12.1$, highlighting the potential importance of this pathway

67 in the genetic etiology of asthma. A crucial role for IL-33 in allergic airway inflammation

68 and bronchial airway hyperresponsiveness has been known since its discovery in

$692005^{3}$. Studies in individuals with asthma and in murine asthma models have identified

70 elevated levels of IL-33 protein in both sera and tissues ${ }^{4,5}$. This cytokine is a potent

71 inducer of type 2 immune responses through its receptor ST2 and has been broadly

72 implicated in other allergic and inflammatory conditions, such as atopic dermatitis,

73 allergic rhinitis, and eosinophilic esophagitis $6,7,8$.

The single nucleotide polymorphisms (SNPs) associated with increased asthma

75 risk at the IL33 GWAS locus reside within a linkage disequilibrium (LD) block in a

76 noncoding genomic segment located $2.3 \mathrm{~kb}$ upstream of the IL33 gene. We therefore

77 posited that variants in this region impact on IL33 expression by altering cis-regulatory

78 element(s) that control quantitative, spatial and/or temporal-specific gene expression. 
79 Previous studies of complex diseases have shown how regulatory variants in promoters

80 and enhancer elements lead to an increased risk of disease through altering the

81 expression of nearby genes $9,10,11,12,13$. In contrast, other types of cis-regulatory

82 elements, including repressors and insulators (also known as enhancer blocking

83 elements), are less understood and characterized than enhancers, but are also likely to

84 be functionally modified by regulatory variants ${ }^{14}$.

Here, we combined genetic fine-mapping using GWAS data sets with functional annotations from relevant tissues to dissect the asthma-associated region upstream of

87 the IL33 gene. We identified a regulatory element harboring SNPs that control IL33 expression. Genotypes at rs1888909, a SNP within this regulatory element, are

89 associated with IL33 expression in ethnically diverse populations, as well as IL-33

90 plasma protein levels. Our study provides functional insights into the role of common

91 regulatory variants at the IL33 locus and illustrate how a causal SNP can exert

92 phenotypic effects by modulating the function of regulatory elements that do not fit into 93 standard definitions of enhancers, insulators, or repressors.

96 Results

97 Defining the IL33 locus asthma-associated critical region

Variants at the IL33 locus have been robustly associated with asthma in GWAS 99 of ethnically-diverse populations ${ }^{2}, 15,16,17,18,19,20$. We first used LD between the most

100 significantly associated SNP in each GWAS (here on referred to as the lead SNP) and 101 other SNPs to define the region harboring potentially causal variants at this locus (Fig. 
102 1a). Five lead SNPs were reported among seven large GWAS, defining an LD block

103 spanning $41 \mathrm{~kb}$ in European ancestry individuals (chr9: 6,172,380-6,213,468, hg19;

104 Supplementary Fig. 1a), which included 21 additional SNPs in LD $\left(r^{2} \geq 0.80\right)$ with at

105 least one of the five lead SNPs (26 total SNPs). Because LD tracts are longer in

106 European genomes compared to African ancestry genomes, we also sought results of

107 GWAS in African Americans to potentially narrow this region. Three multi-ancestry

108 GWAS 16, 18, 19 included African Americans, but only one ${ }^{16}$ provided GWAS results

109 separately by ancestry. In that study, the lead GWAS SNP rs 1888909 differed from the

110 lead SNPs in the European ancestry (rs1342326 ${ }^{15}$, rs928413 ${ }^{17}, \mathrm{rs} 7848215^{20}$ and

111 rs992969²) or combined multi-ancestry (rs2381416 ${ }^{16}$ and rs992969 ${ }^{18,19}$ ) GWAS.

112 Because there was so little LD in this region in African Americans, we used an $r^{2} \geq 0.40$

113 to define LD. SNPs in LD with rs1888909 at $r^{2} \geq 0.4$ in African Americans defined a

114 region of $20 \mathrm{~kb}$ (chr9: 6,188,124-6,209,099, hg19; Supplementary Fig. 1b and

115 Supplementary Table 1) that excluded two lead SNPs (rs928413 ${ }^{17}$ and rs7848215 ${ }^{20}$ ).

116 To gain more formal statistical support for this interval, we next used a Bayesian

117 method, Sum of Single Effects (SuSiE) ${ }^{21}$, to fine-map the associated region and identify

118 credible sets (CS) of SNPs at the IL33 locus with high probabilities of being causal.

119 Within each CS, variants are assigned posterior inclusion probabilities (PIPs), with

120 higher PIPs reflecting higher probabilities of being causal and the sum of all PIPs within

121 a CS always equaling 1. Using all SNPs at this locus from a GWAS of childhood onset

122 asthma ${ }^{2}$ in British white subjects from the UK Biobank ${ }^{22}$, we identified three CSs

123 (Fig.1b). CS1 contained variants with the highest PIPs and included six SNPs, all

124 among those defined by LD with the lead GWAS SNPs, defining a $12.7 \mathrm{~kb}$ region (chr9: 
$1256,197,392-6,210,099$; hg19) approximately $22 \mathrm{~kb}$ upstream of the transcriptional start 126 site of the IL33 gene (Fig. 1a and Supplementary Table 2). The three CS1 SNPs with 127 the highest PIPs included the lead SNPs in two multi-ancestry GWAS (rs992969; PIP 128 0.391), the lead SNP in the African American GWAS $^{16}$ (rs1888909; PIP 0.209), and a 129 SNP in LD with the lead SNPs (rs3939286; PIP 0.205). We note that two other CSs of 13025 SNPs (CS2) and 28 SNPs (CS3) were identified by SuSiE, suggesting additional, 131 independent regions potentially regulating the expression of IL33 or other genes.

132 Next, we used Roadmap Epigenome ${ }^{23}$ and ENCODE ${ }^{24}$ data to annotate the 133 regulatory landscape of the more inclusive $20 \mathrm{~kb}$ asthma-associated interval defined by

134 LD. This segment was enriched with chromatin marks and DNase hypersensitive sites

135 (Fig. 1c), suggestive of regulatory potential in multiple cell types. We also identified two 136 CTCF sites within $2 \mathrm{~kb}$ of each other with evidence of CTCF binding in multiple cell lines

137 (84 cell lines have CTCF binding to site 1 and 142 have CTCF binding to site 2 out 194 138 ENCODE-3 lines). In addition, the cohesin complex RAD21-SMC-3 subunits and zinc139 finger proteins such as ZNF384 and ZNF143 also bind to this region (Fig. 1d). Binding 140 of this multi-subunit complex along with CTCF can provide sequence specificity for 141 chromatin looping to promoters or have insulator functions ${ }^{25,26}$.

142 Interestingly, the three lead GWAS SNPs (rs1342326 ${ }^{15}$, rs2381416 ${ }^{16}$ and 143 rs9929692, 18, 19) in the LD-defined $20 \mathrm{~kb}$ region did not overlap with regions of open

144 chromatin or transcription factor binding; one, rs9929692, 18, 19, mapped within 145 heterochromatin (thick blue horizontal bar) in virtually every ENCODE cell line 146 (Supplementary Fig. 2). Heterochromatin is a highly compacted region in the genome 147 and not actively involved in gene regulation. For these reasons, it is not likely any of 
148 these three lead SNPs within the LD-defined region are causative of the asthma

149 association. In contrast, five SNPs in high LD with the asthma-associated lead SNPs,

150 including two from CS1 and including the lead African American GWAS SNP

151 (rs1888909), overlapped with a region of open chromatin and CTCF, cohesin and ZNF

152 binding, delineating a discrete $5 \mathrm{~kb}$ region (chr9: 6,194,500-6,199,500, hg19) (Fig. 1c-

153 d). Collectively, these data reveal a $5 \mathrm{~kb}$ region that harbors both asthma-associated

154 SNPs and marks of regulatory activity that could modulate IL33 expression through

155 long-range interactions. 
a

b
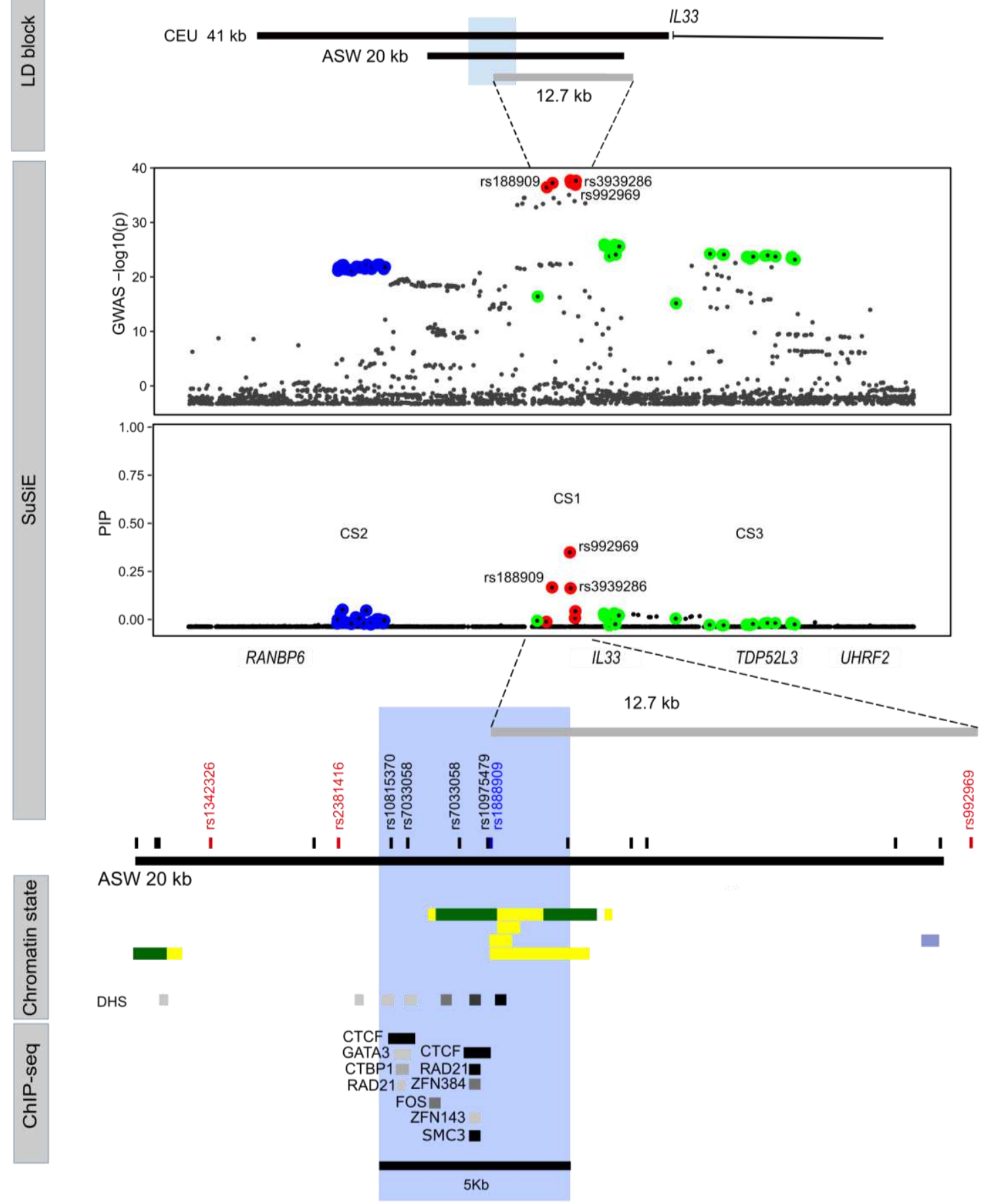

157 Figure 1. Epigenetic characterization of the asthma-associated critical region in the IL33 locus. a

158 Schematic organization of the IL33 gene and the asthma-associated region (black bars) of European

159 ancestry (CEU 41 kb, chr9: 6,172,380-6,213,468; hg19) and African ancestry (ASW 20 kb, chr9:

160 6,188,124-6,209,099; hg19) positioned upstream of exon 1. b Significance of SNP association in the

161 GWAS $^{2}$ (top) and fine mapping results (bottom) for each variant at the IL33 locus (bottom). Colors 
162 indicate each credible set (CS) identified. CS1: red, CS2 blue; CS3 green. CS1 (rs992969, rs1888909, 163 rs3939286) defines a region of $12.7 \mathrm{~kb}$ (chr9: 6,197,392 6,210,099; hg19). c Position of the lead GWAS

164 SNPs (in red) and additional SNPs in high LD ( $\left.r^{2} \geq 0.8\right)$ with the lead SNPs (in black) within the ASW $20 \mathrm{~kb}$

165 LD region. The lead SNP rs1888909 in African ancestry is shown in blue. Chromatin states from

166 Roadmap Epigenomics Project showing regions with potential regulatory activity. Yellow: active

167 enhancer; green: transcribed sequence; blue: heterochromatin. DNase hypersensitive (DHS) sites

168 indicating open chromatin regions are shown. Tissues (from the top): E096 Lung primary HMM; E095 Left

169 ventricle primary HMM; E116 GM128781 Lymphoblastoid cell primary HMM; E122 HUVEC Umbilical Vein

170 Endothelial Primary Cells Primary HMM d ChIP-seq data from ENCODE-3 cell lines (338 factors; 130 cell

171 types) showing co-binding of CTCF, RAD2, ZFNs and SMC-3 at the $5 \mathrm{~kb}$ interval (blue shaded region;

172 chr9: 6,194,500-6,199,500; hg19).

173

174

175 Regulatory properties of a $5 \mathrm{~kb}$ region upstream of IL33

Having identified a region of interest, we sought to determine its impact on IL33

177 expression. Because IL33 is expressed in multiple tissues, we used an in vivo model

178 system to assay for the spatial regulatory properties of this $5 \mathrm{~kb}$ region. However, the

179 lack of evolutionary conservation at the locus between human and mouse

180 (Supplementary Fig. 3) required the creation of a "humanized" transgenic mouse model.

181 For this, we used a human Bacterial Artificial Chromosome (BAC - clone RP11-

$182725 \mathrm{~F} 15)$, approximately $166 \mathrm{~kb}$ long, spanning the coding region of the IL33 gene and

183 its upstream sequences, including the $20 \mathrm{~kb}$ asthma-associated region. We recombined

184 a cassette containing a E2-Crimson reporter with a 3' stop codon into exon 2, in frame

185 with the IL33 start codon (ATG). Any IL33 regulatory regions within this BAC would

186 drive E2-Crimson expression, mimicking IL33 endogenous spatio-temporal expression

187 patterns. Also, to directly test the regulatory impact of the $5 \mathrm{~kb}$ region, we selectively

188 deleted this asthma-associated DNA segment from the full BAC and assessed the 
189 resultant IL33 expression in vivo, in mice harboring either the full length or the $5 \mathrm{~kb}$ 190 deletion BAC (Fig. 2a).

191 Immunofluorescence staining of peripheral lymph node from full BAC transgenic 192 mice ( $\mathrm{h} / \mathrm{L} 33^{\text {Crim }} \mathrm{BAC}$ ) showed that E2-Crimson fluorescent protein is highly expressed in 193 this tissue (Fig. 2b, upper panel and Supplementary Fig. 4a). Strikingly, constitutive 194 expression of the E2-Crimson reporter was co-localized with the endothelial cell marker 195 CD31 and observed in high endothelial venule (HEV) cells in mouse lymph nodes. This 196 observation validates the species-specificity of the E2-Crimson expression, as previous 197 studies report that while IL-33 is produced by HEV in humans, it is not found in murine $198 \mathrm{HEV}^{27}$. In contrast, deletion of the $5 \mathrm{~kb}$ region in the reporter BAC ( $\left.\mathrm{h} / 233^{\mathrm{Crim}} \mathrm{BAC} 5 \mathrm{kdel}\right)$ 199 significantly depleted E2-Crimson immunostaining in lymph nodes compared to the 200 staining observed in the full BAC mice (Fig. 2b, lower panel and Supplementary Fig. 201 4b). The $5 \mathrm{~kb}$ deletion also significantly reduced E2-Crimson mRNA expression in heart 202 and lung in three out of four independent lines (Fig. 2c). These results show that the 203 BAC encodes human-specific regulatory patterns in vivo and demonstrate the 204 importance of the $5 \mathrm{~kb}$ noncoding segment for proper IL33 expression. 
a

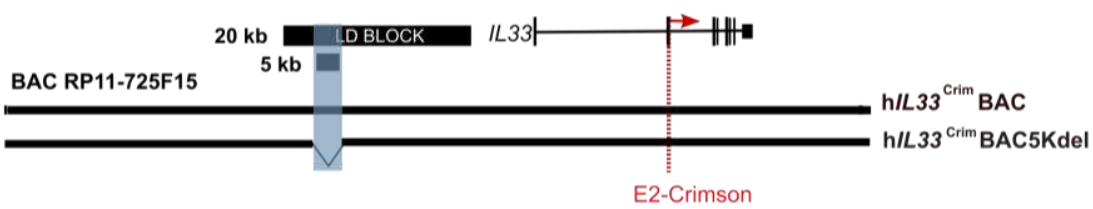

b

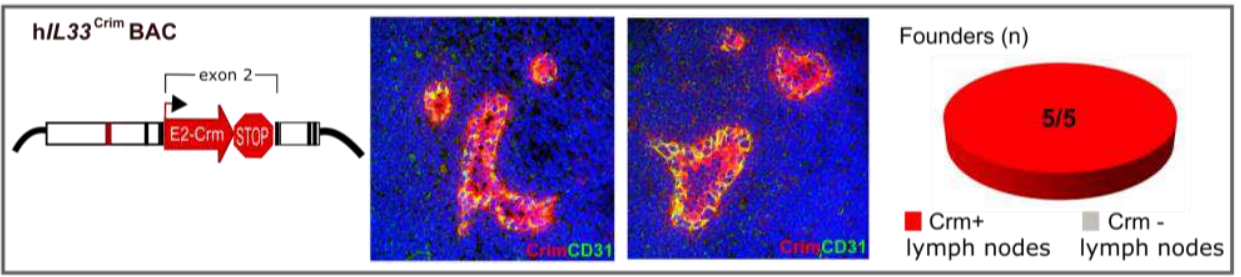

h/L33 ${ }^{\text {Crim }}$ BAC5Kdel
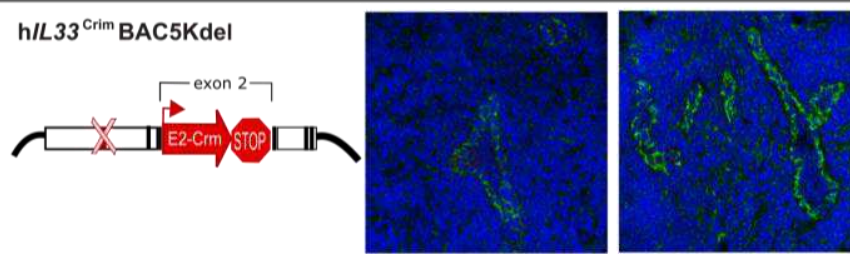

lymph nodes

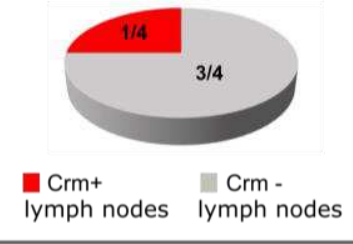

C

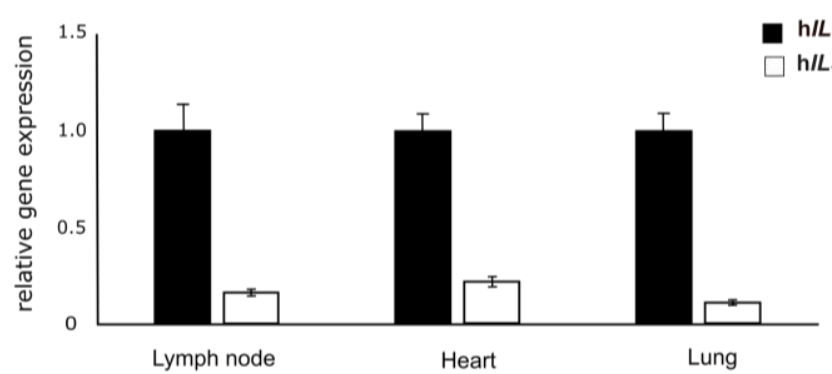

212 Figure 2. The IL33-containing BAC in transgenic mice encodes human-specific regulatory patterns

213 and demonstrates the importance of the $\mathbf{5} \mathbf{~ k b}$ noncoding segment for proper IL33 expression. a

214 Schematic of human BAC clone RP11-725F15 (166 kb) spanning the entire coding region of IL33 and its

215 upstream region including the $20 \mathrm{~kb}$ asthma-associated interval and the $5 \mathrm{~kb}$ region of interest shaded in

216 blue (black bars). To produce a human IL33 reporter strain, a cassette containing E2-Crimson with a stop

217 sequence was inserted into exon 2, in frame with the IL33 translational start site (red dotted line).

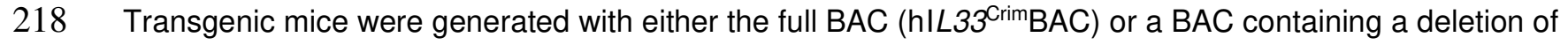

219 the $5 \mathrm{~kb}$ interval within the LD block (h/L33 Crim BAC5Kdel). b Immunofluorescence staining of mouse

220 peripheral lymph node sections of E2-Crimson in h/L33 ${ }^{\text {Crim }}$ BAC mice (upper panels) or h/L33 Crim

221 BAC5Kdel (lower panels). Representative founder BAC transgenic lines are shown. Sections were

222 stained with anti-E2-Crimson (red) and the mouse endothelial cell marker CD31 (green). Hoechst staining

223 for nuclei is in blue. c qPCR analysis of E2-Crimson mRNA obtained from lymph node (LN), heart and

224 lung from both BAC strains is shown 


\section{Long-range chromatin interactions in the IL33 locus}

226 We next assessed the physical interactions between the asthma-associated

227 region and the IL33 promoters by performing circular chromosome conformation

228 capture followed by high-throughput sequencing (4C-seq) in cells obtained from

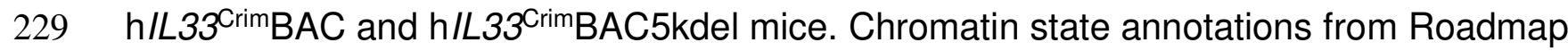

230 Epigenome suggest the presence of distal regulatory elements, such as enhancers

231 (yellow bars) distributed across the human BAC region containing the IL33 gene and its

232 upstream non-coding DNA (Fig. 3a).

233 We positioned the viewpoint directly upstream of the $5 \mathrm{~kb}$ region in order to

234 capture interactions between this genomic region and the entire adjacent IL33 locus

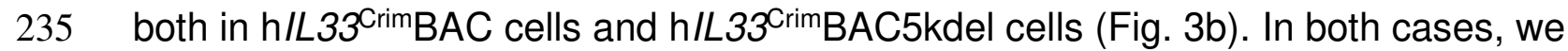

236 observed interactions between the viewpoint and several potential regulatory regions

237 located upstream and downstream (gray arcs), including the less frequently used

238 promoter of the long transcript as well as the predominantly used promoter for the short

239 transcript of IL33 (blue arcs). Deletion of the $5 \mathrm{~kb}$ fragment reduced the interactions at

240 the IL33 locus compared to those observed using the full BAC, including the loss of the

241 interaction with the short transcript promoter, confirming the necessity of this region for

242 proper IL33 regulation and explaining the loss of E2-Crimson expression in

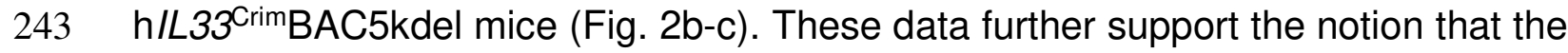

244 asthma-associated LD region harbors regulatory elements that physically interact with

245 IL33 to regulate its expression. 


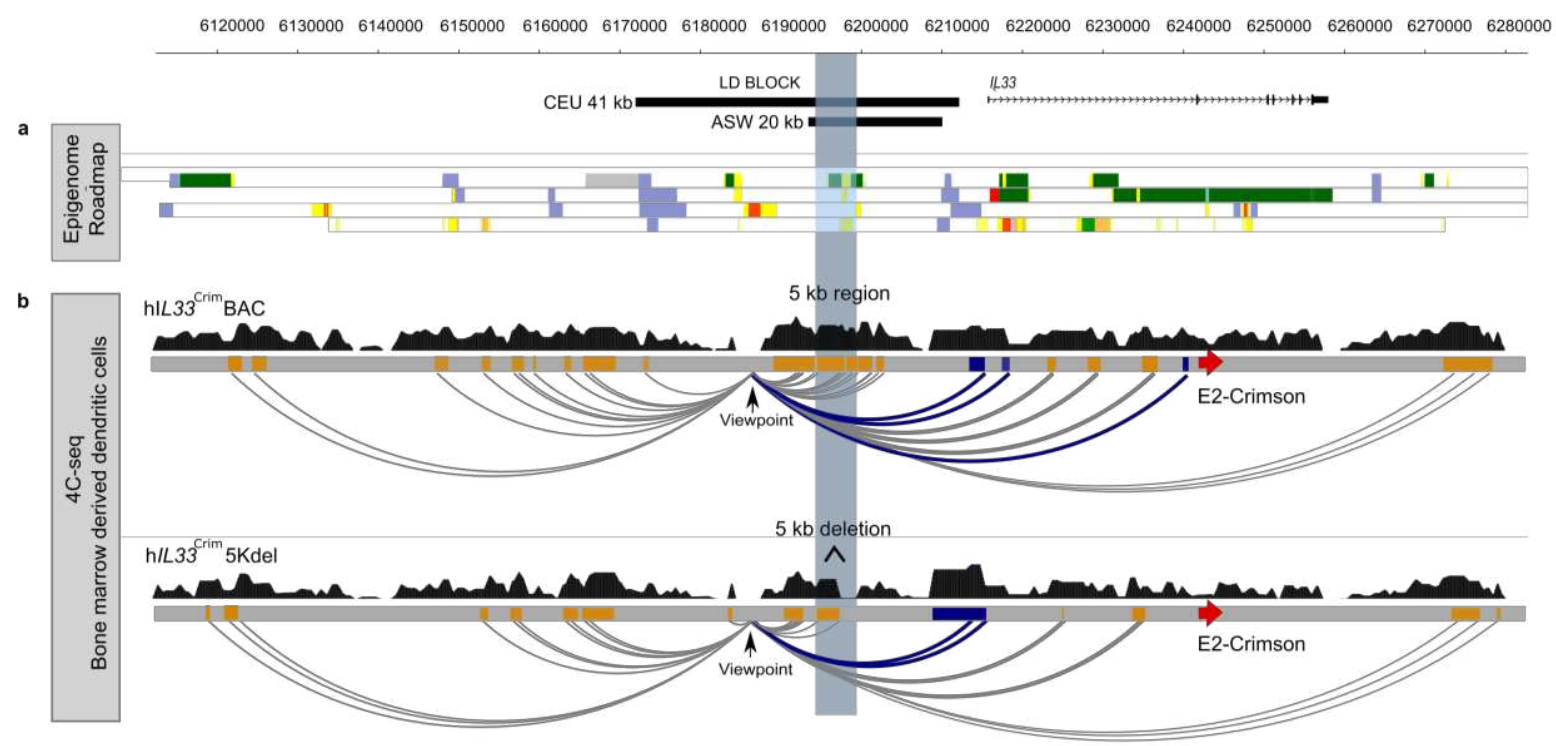

247 Figure 3. Interaction maps show looping of the asthma-associated region to the IL33 promoter. a

248 Roadmap Epigenome data from heart (E095 Left ventricle primary HMM), lung (E096 Lung primary

249 HMM), LCL (E116 GM128781 Lymphoblastoid cell primary HMM) and endothelial cells (E122 HUVEC

250 Umbilical Vein Endothelial Primary Cells Primary HM). Chromatin state assignments are indicated as:

251 transcribed, green; active enhancer, yellow; active promoter, red; repressed, gray; heterochromatin, blue.

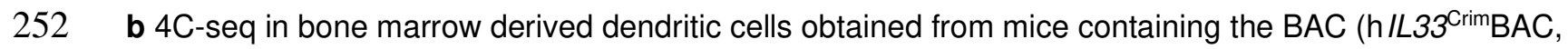

253 top) and a deletion of the $5 \mathrm{~kb}$ interval within the LD block (h/L33 Crim BAC5Kdel, bottom). Gray bars

254 correspond to a schematic representation of the human BAC showing the E2-Crimson reporter insertion

255 site on exon 2 of the IL33 gene (red arrow) and the asthma associated $5 \mathrm{~kb}$ region (blue shaded box). In

256 both experiments, reads were mapped to the coordinates corresponding to the human BAC (chr9:

$2576,112,733-6,279,294$; hg19) and peaks shown in dark blue and orange represent the promoter and distal

258 elements, respectively. Arcs depict interactions from the viewpoint located upstream the $5 \mathrm{~kb}$ region.

259 Interactions between the LD region, near to the $5 \mathrm{~kb}$ region (viewpoint), and the IL33 promoters are noted

260 in blue

\section{Asthma-associated SNPs modify regulatory properties of the $5 \mathbf{k b}$ region}

To functionally characterize the regulatory impact of allelic variants of asthmareporter assays. Because this region overlaps with chromatin states suggestive of 
267 enhancer function in some cell types (Fig. 1c), and because its deletion in the BAC

268 resulted in a generalized loss of reporter expression in vivo, we hypothesized that the 5

$269 \mathrm{~kb}$ region corresponds to an enhancer. To test the regulatory potential of this DNA

270 segment in vitro, we cloned both the risk and non-risk haplotypes of the $5 \mathrm{~kb}$ fragment in

271 a luciferase vector and transfected them in human cell lines. We used an immortalized

272 human aortic endothelial cell line (TeloHAEC) and K562, as the ENCODE project

273 chromatin data annotate this region as a putative enhancer in endothelial and blood

274 cells. We failed to detect enhancer activity of either haplotype in the $5 \mathrm{~kb}$ fragment

275 (Supplementary Fig. 5).

276 Interestingly, upon close inspection of ENCODE data we notice that this $5 \mathrm{~kb}$

277 region is not bound by transcription factors usually associated with enhancer activity,

278 including transcriptional activators, repressors, or RNA Poll. Rather, it is bound by both

279 CTCF and subunits of the cohesin complex across multiple cell lines (Fig. 1d). Both

280 CTCF and cohesin are key determinants of chromatin loop formation and stabilization,

281 including the positioning of regulatory elements close to the promoters of their target

282 genes, or serving as insulators, with enhancer blocking properties.

283 To test the enhancer blocking properties of the $5 \mathrm{~kb}$ fragment, we first used an in

284 vitro luciferase-based assay in which candidate sequences are cloned between a strong

285 promoter (SV40) and a strong enhancer (HS2 element in human beta-globin LCR) ${ }^{28}$.

286 Luciferase expression is driven by the enhancer and promoter elements, and a

287 decrease in luciferase activity would be interpreted as enhancer blocking activity, with

288 the enhancer not able to loop to the adjacent SV40 promoter. When the $5 \mathrm{~kb}$ region was

289 cloned into this vector we observed prominent enhancer-blocking activity (Fig. 4a). 
290 Significant differences were also observed between fragments containing either the risk

291 or non-risk alleles for the five SNPs, with the risk allele showing weakened enhancer

292 blocking activity (Fig. 4b; $\mathrm{p}=0.001$ ). As a control, we used a DNA sequence located 60

$293 \mathrm{~kb}$ upstream of IL33 and devoid of any epigenetic marks of active chromatin and with no

294 evidence of CTCF or cohesin binding. This control sequence had no significant impact

295 on the reporter gene expression (Fig. 4a).

To test the this enhancer blocking property in vivo we used a zebrafish reporter

297 assay $^{29}$. The $5 \mathrm{~kb}$ region was cloned in a reporter cassette containing Green

298 Fluorescent Protein (GFP) driven by a cardiac actin promoter and a midbrain enhancer.

299 An enhancer blocking element cloned in this vector would restrict the access of the

300 midbrain enhancer to the GFP reporter gene, while the ability of the actin promoter to

301 activate GFP in skeletal muscle and somites would be maintained (Fig. 4c). In these

302 experiments, the $5 \mathrm{~kb}$ region led to a decreased mid-brain-specific GFP signal when

303 compared to the control sequence, which displayed no enhancer blocking activity (Fig.

$3044 d ; p<0.001)$. Similar to the data in the in vitro luciferase experiments, there was a

305 significant difference in GFP activity between fragments containing the risk or non-risk

306 alleles ( $p=0.041)$, with the risk alleles resulting in reduced enhancer blocking activity.

307 Taken together, our data provide evidence that the $5 \mathrm{~kb}$ region has enhancer blocking

308 properties in vitro and in vivo, and that alleles of asthma-associated SNPs within this

309 region are able to modulate this property.

$310 \quad$ The $5 \mathrm{~kb}$ fragment contains two CTCF sites within $2 \mathrm{~kb}$ of each other and 5

311 asthma-associated SNPs. To determine the impact of the SNPs on its regulatory

312 function, we cloned a smaller $1 \mathrm{~kb}$ fragment, including one CTCF site and the SNPs 
313 rs10975479 and rs1888909. These SNPs are located $15 \mathrm{bp}$ apart from each other and

314 display the highest LD $\left(r^{2}\right)$ with the tag SNP rs992969 (0.56 and0.96, in CEU

315 respectively). This segment of the $5 \mathrm{~kb}$ region overlaps with the minimal critical region

316 predicted by fine-mapping by SuSie (Fig. 1a). This shorter fragment still showed

317 prominent enhancer blocking activity in vitro, in luciferase assays (Fig. 4e).

318 Furthermore, deletion of $400 \mathrm{bp}$ within the $1 \mathrm{~kb}$ element, harboring the SNPs

319 rs10975479 and rs1888909, abrogated the enhancer blocking activity of this fragment.

320 Importantly, this $400 \mathrm{bp}$ deletion does not span the CTCF binding site within the $1 \mathrm{~kb}$

321 fragment. Testing these fragments in our in vivo zebrafish transgenic reporter assay

322 confirmed the $1 \mathrm{~kb}$ fragment enhancer blocking activity and the functional importance of

323 one or both SNPs within the 400 bp deleted region in regulating GFP expression (Fig.

324 4f). Together, these data characterize a regulatory region upstream of the IL33 locus

325 and implicate the asthma-associated SNPs rs10975479 and rs1888909 in regulating

326 IL33 expression.

327 
a

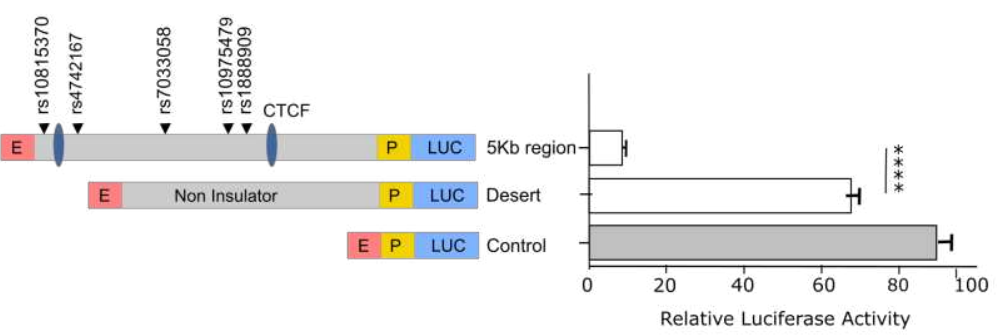

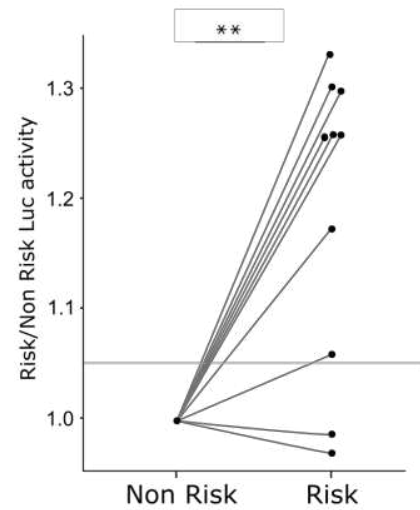

c
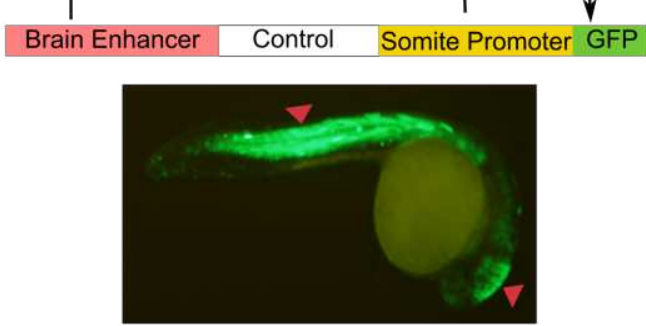

Brain Enhancer $5 \mathrm{~kb}$ region Somite Promoter GFP

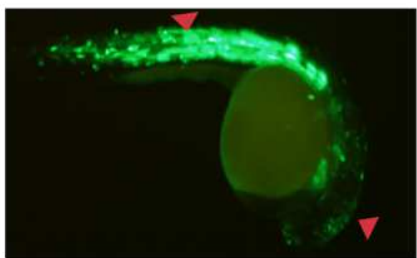

e

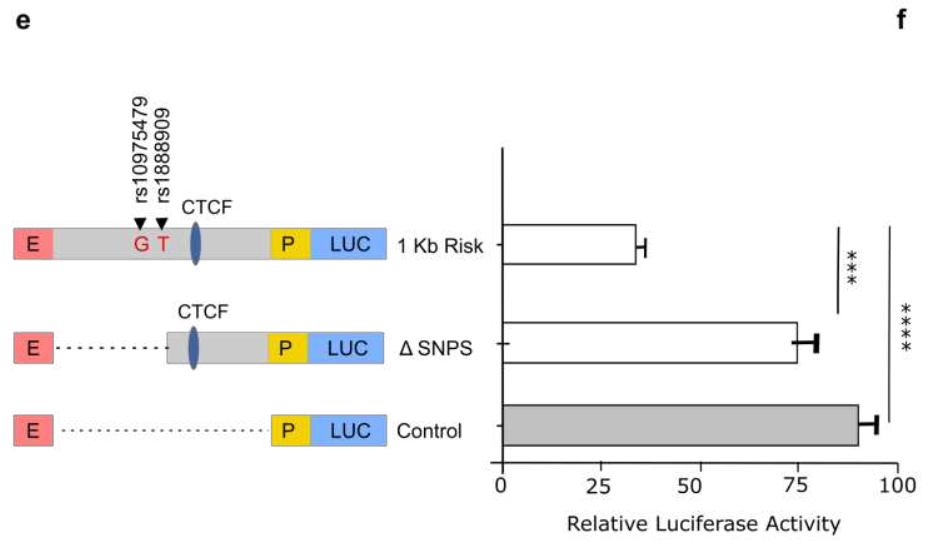

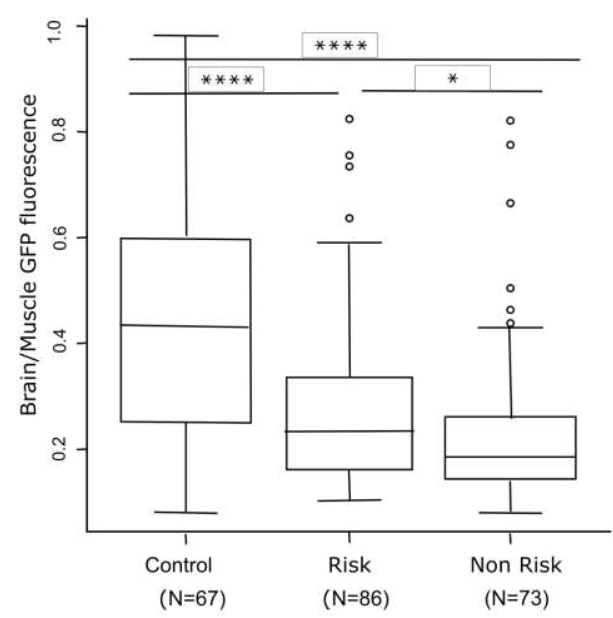

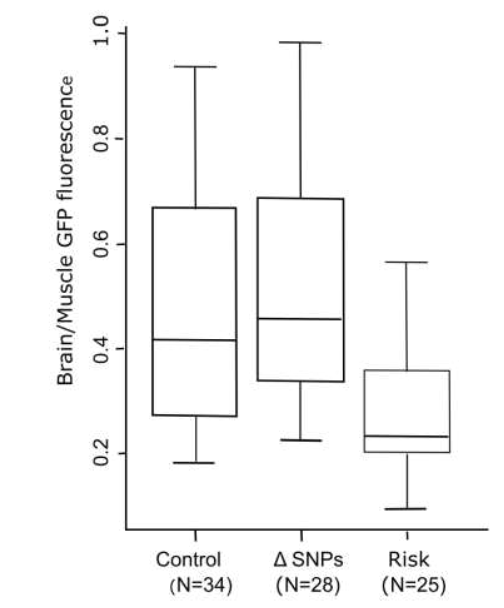

329 Figure 4. Impact of the asthma-associated variants in the regulatory property of the $5 \mathrm{~kb}$ region. a

330 In vitro transgenic reporter assay. Luciferase based enhancer barrier assay using 5 kb constructs (chr9:

$3316,194,500-6,199,500$; hg19) that were cloned between HS2 enhancer (E) and SV40 promoter (P) 
sequences. SNPs in the construct are noted (black arrowheads). Results are from 3 independent experiments. ${ }^{* * * *} p<0.0001$, unpaired t-test. $\mathbf{b}$ Luciferase activity values of the risk construct is shown as fold change over the activity obtained in the non-risk sequence. Results shown represent data from 10 independent experiments. ${ }^{* *} \mathrm{p}=0.0014$, two-way ANOVA. c In vivo zebrafish transgenic reporter assay. Green fluorescent protein (GFP) expression 24 hours post fertilization (hpf) in mosaic F0 embryos injected with vectors containing a control sequence (top panel) or $5 \mathrm{~kb}$ interval sequence (bottom panel). d Comparison between $5 \mathrm{~kb}$ constructs containing risk or non-risk alleles for enhancer blocking property. Data is presented as midbrain/somites EGFP intensity ratio compared with empty gateway vector which has no enhancer blocking activity. ${ }^{* \star *} \mathrm{p}=1.1 \mathrm{e}-5 ;{ }^{*} \mathrm{p}=0.041$, ANOVA pairwise T-test. e $1 \mathrm{~kb}$ DNA fragment (chr9: 6,197,000-6,197,917; hg19) containing the risk alleles for asthma variants rs 10975479 and rs1888909 or a 400bp deletion in the region harboring those SNPs (chr9: 6,197,399-6,197,914; hg19) were assayed for luciferase reporter activity in K562 cells. Results are representative of 3 independent

344 experiments ${ }^{* *} \mathrm{p}=0.0005$, unpaired $\mathrm{t}$-test. $\mathrm{f}$ Zebrafish reporter assay comparing the $1 \mathrm{~kb}$ DNA fragment 345 and fragment with the $400 \mathrm{bp}$ deletion $(\Delta \mathrm{SNP})$. Data are presented as in (d).

\section{Differential binding of OCT-1 to allelic variants of rs1888909} hypothesized that the risk alleles of rs1888909 may alter transcription factor binding,

350 resulting in altered regulatory activity and IL33 expression. Although not predicted to be

351 a causal SNP (Fig. 1b), we also studied rs10975479 (G/A) as it is only 15 bp away from

352 rs1888909. We performed an electrophoretic mobility shift assay (EMSA) using small

353 labeled DNA probes and unlabeled competitors spanning four different combinations of

354 the risk or non-risk alleles for variants rs10975479 (G/A) and rs1888909 (T/C). Upon

355 incubation with nuclear extract, we observed differences in binding patterns between

356 the risk (G-T) and non-risk (A-C) probes, suggesting that different transcription factor

357 binding complexes are formed in the presence of these two alleles (Fig. 5a). The major

358 changes in binding are observed with both probes carrying the rs1888909 (T) allele,

359 thereby indicating that the difference is driven by the risk allele T of this SNP. These 
360 data demonstrate that risk allele (T) of the asthma-associated SNP rs1888909 alters

361 protein binding properties, possibly influencing IL33 expression.

362 To identify nuclear proteins that bind to the rs1888909 (T) vs rs1888909 (C)

363 probes, we isolated 3 bands from the non-risk rs 1888909 (C) lane, and 2 bands from

364 the risk rs1888909 (T) lane for mass spectrometry analysis (Supplementary Fig. 6). As

365 a control, we isolated the same regions in the lane where the lysate had been incubated

366 with a cold probe competitor. First, we filtered out the proteins that were also found in

367 the control lane and nuclear proteins that aren't known to bind to DNA (Supplementary

368 Table 3). We identified three transcription factors with known DNA binding motifs bound

369 only to the non-risk probe (NFE2, TFCP2 and FOXL2) and four transcription factors

370 bound only to the risk probe (POU2F1, FOXP1, STAT3 and STAT5b). We then used the

371 UniPROBE protein array database ${ }^{30}$ to select the transcription factors known to bind to

372 at least one octamer containing a risk or non-risk allele. This analysis resulted in one

373 transcription factor: OCT-1 (POU2F1), which bound to the EMSA probe and was

374 differentially bound to the risk allele. OCT-1 was selected as our primary candidate for

375 further investigation.

376 To confirm OCT-1-specific binding to the risk probe we first used a cold

377 competition assay and demonstrated that the band specifically competed with an OCT-1

378 canonical DNA binding motif, but not with a mutated oligonucleotide (Fig. 5b, left panel).

379 Conversely, binding to the non-risk A-C probe was not competed by the consensus or

380 mutated OCT-1 oligonucleotides, demonstrating specificity of OCT-1 association with

381 only the risk allele (Fig. 5b, right panel). Further, an OCT-1 specific antibody

382 supershifted the nuclear complex formed with the rs1888909 (T) probe (Fig. 5c). We 
383 were able to visualize this shift independently of the order of incubation of the nuclear

384 extract with probe or antibody, suggesting robust protein binding. We then performed

385 chromatin immunoprecipitation (ChIP) followed by qPCR to demonstrate enrichment of

386 OCT-1 binding to the DNA region containing the rs18889099 (T) (Fig. 5d). These

387 experiments provide strong evidence that OCT-1 binds differentially to the risk allele

388 and directs the formation of differential allelic nuclear complexes at the rs1888909

389 variant.

390 
a

rs10975479 - rs1888909
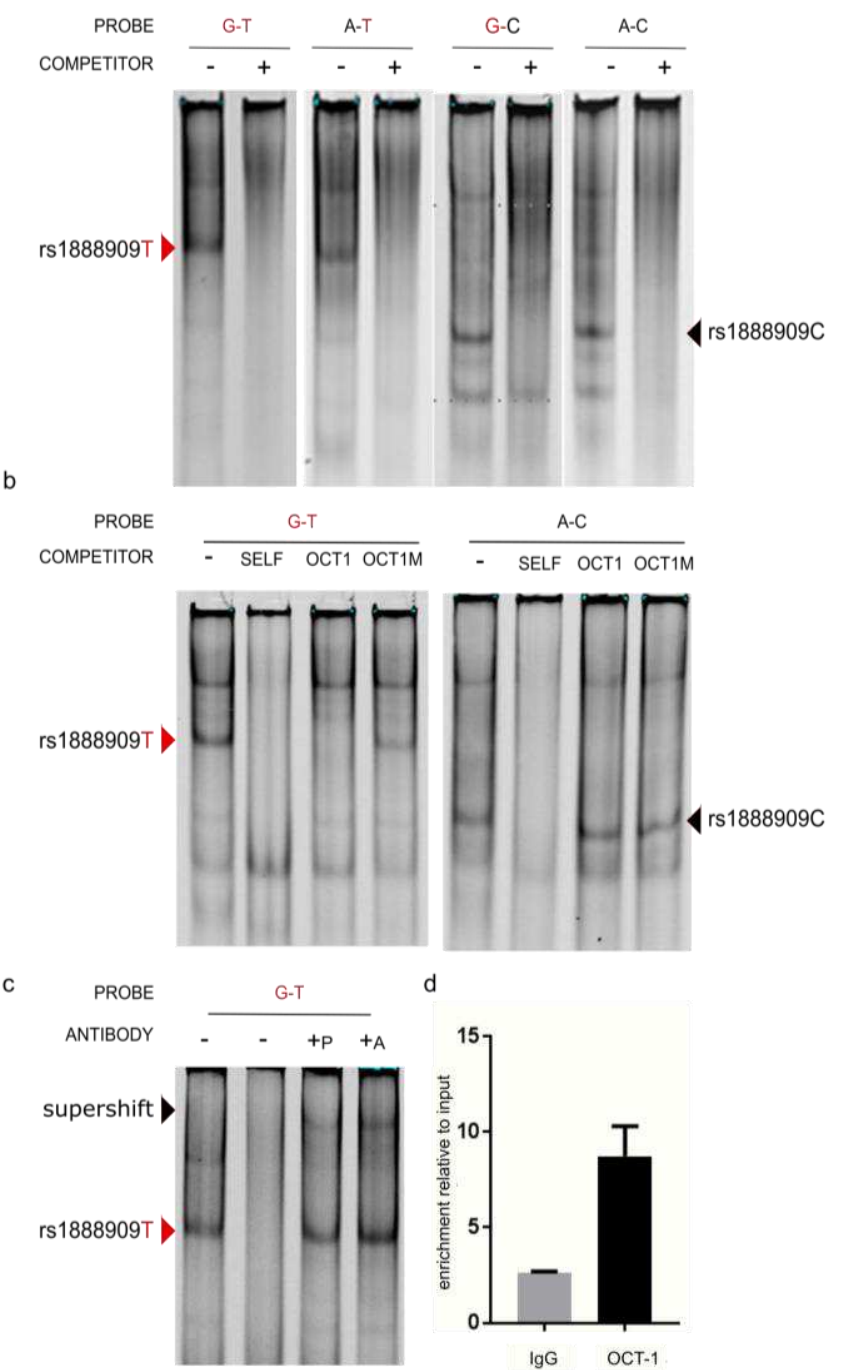

392 Figure 5. Regulatory region containing the risk allele rs1888909T selectively binds OCT-1.

393 a Radiolabeled probes carrying the risk (in red) and/or non-risk sequences for SNPs rs 10975479 and

394 rs1888909 were incubated with nuclear extract obtained from K562 cells. Different complexes formed by

395 rs1888909 are marked by red or black arrows. b Cold competition assay with OCT-1 consensus (OCT1)

396 or mutated OCT-1 (OCT1M) oligonucleotides. EMSA probes and oligo competitor (100x molar excess)

397 are noted above each gel. c Supershift complex formation with addition of anti-OCT-1 antibody as

398 indicated by the red arrow. $+\mathrm{P}$ indicates addition of probe with nuclear extract, followed by incubation with

399 antibody. $+\mathrm{A}$ indicates incubation of extract with antibody followed by addition of probe, $+\mathrm{P}$ indicated

400 incubation of extract with probe followed by antibody. $\mathbf{d}$ Chromatin immunoprecipitation of H292

401 chromatin with anti-OCT-1 antibody shows percent enrichment to input chromatin compared to control

402 IgG antibody. Results are representative of 3 independent experiment 
Finally, we tested the functional consequences of the GWAS SNPs rs 1888909 , rs10975479 and rs992969 on IL33 mRNA and IL-33 protein abundance. The first two

406 SNPs are within the $5 \mathrm{~kb}$ region. The latter, rs992969, is located outside of this region, 407 but was previously reported to be associated with IL33 expression in bronchial epithelial 408 cells from primarily non-Hispanic white subjects ${ }^{31}$. SNP rs992969 is in high LD with 409 rs1888909 in European ancestry populations ( $r^{2}=1$ in CEU), but less so in African 410 American populations ( $r^{2}=0.45$ in ASW), and in low LD with $r s 10975479$ in both 411 populations $\left(r^{2}=0.56\right.$ and 0.13 , respectively) (Supplementary Figure 1 and

412 Supplementary Table 1). We used RNA-seq data from endobronchial brushings

413 obtained from 124 asthmatic and non-asthmatic adult subjects, mostly of European

414 ancestry (Fig. 6a, upper panel), and from nasal epithelial cell brushings from 189

415 African American children from high risk asthma families (Fig. 6a, lower panel). In both

416 populations, carriers of one or two copies of the rs1888909 (T) risk allele had

417 significantly higher IL33 transcript levels compared to non-carriers of this allele.

418 Genotypes at rs992969 were more modestly associated with transcript levels and only 419 significant in the African American samples after correcting for multiple (3) tests; while 420 rs10975479 was not associated with IL33 transcript abundance in either sample (Fig.

421 6a, Supplementary Table 4). These results are consistent with the EMSA data

422 suggesting that allelic variants of rs1888909 result in differential protein binding. The 423 stronger effects of rs 1888909 on IL33 expression in both populations suggest that 424 rs1888909 is the causal variant in this region and that associations with other variants 
425 (such as rs992969) in GWAS and in gene expression studies were due to LD with the 426 causal variant.

427 We observed similar patterns of association in studies of IL-33 protein levels in 428 plasma from 30 children $^{32}$ of European ancestry (Fig. 6b). Children who carried the 429 asthma risk alleles at rs 1888909 or rs992969 had more IL-33 protein compared to 430 children not carrying these alleles. There was no association between genotype at SNP 431 rs10975479 and IL-33 protein levels.

432 The aggregate of our mouse transgenic assays, in vivo and in vitro reporter 433 assays, and EMSA collectively supported a role for rs 1888909 as a causal variant for

434 the association with IL33 expression. The associations between the risk alleles and 435 increased IL33 transcript levels and IL-33 protein abundance further support a role for 436 this region in regulating the $I L 33$ gene and point to rs 1888909 as the causal variant in 437 this region, corroborating predictions from our in vitro and in vivo studies. 
a
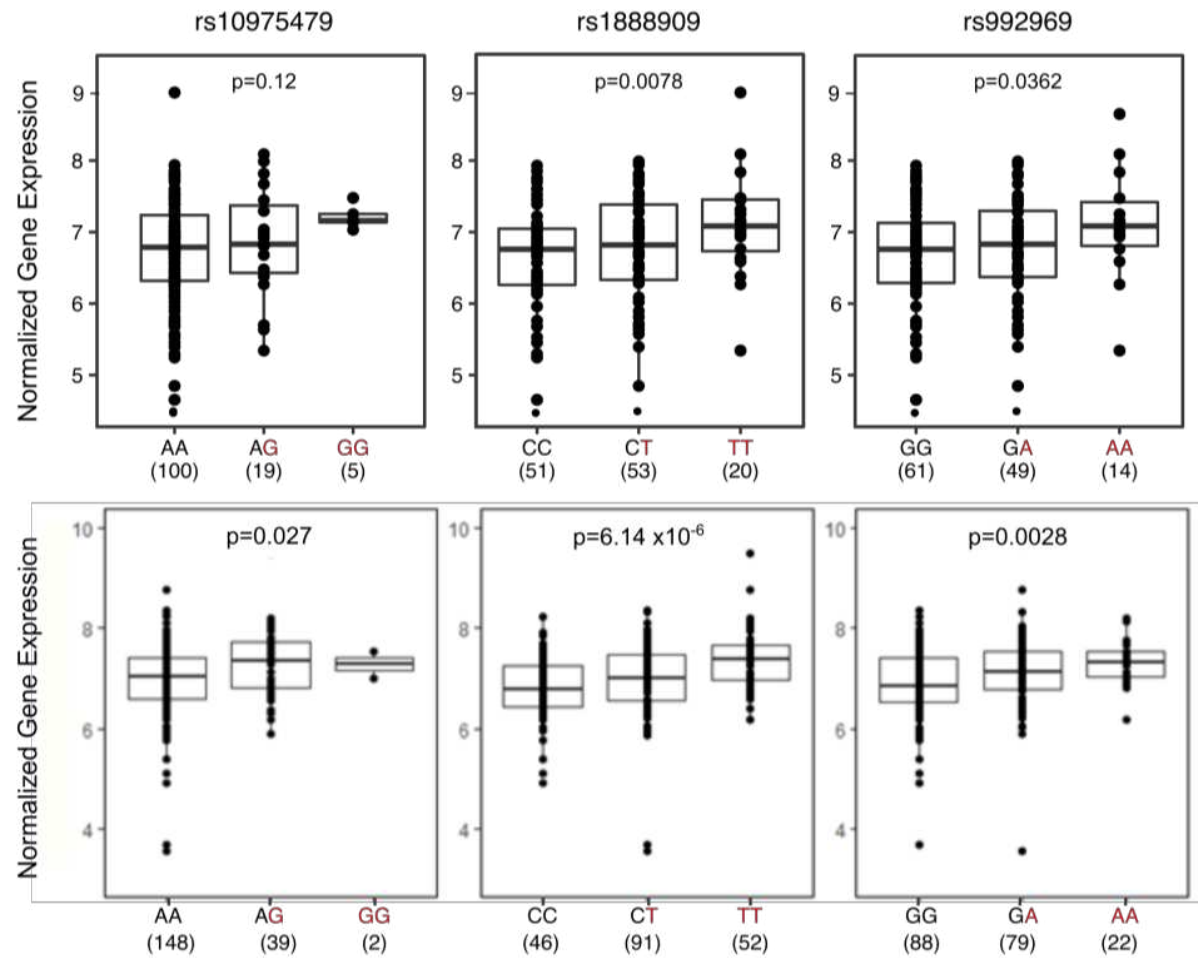

b
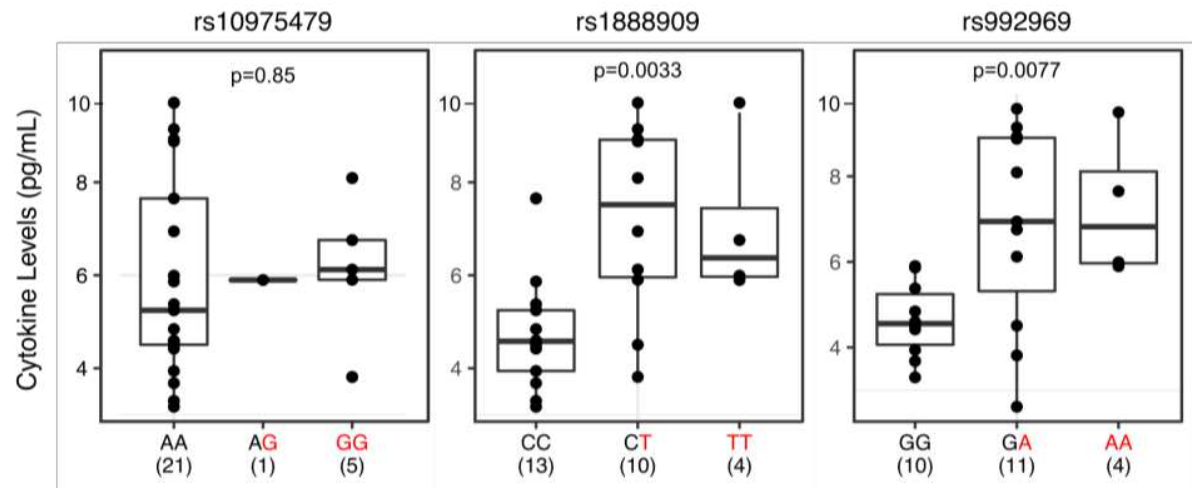

Figure 6. The rs1888909 (T) and rs992969 (A) alleles are associated with increased IL33 expression and IL-33 protein levels. a Comparison of IL33 expression between genotypes for SNPs rs10975479, rs1888909 and rs992969 from endobronchial brushings from 124 asthmatic and non-asthmatic adult subjects, mostly of European ancestry (upper panels) and nasal epithelial cells from 189 African American children from high risk asthma families (lower panels). b Comparison of IL-33 cytokine levels between genotypes for SNPs rs10975479 ( $n=26)$, rs1888909 ( $n=27)$ and rs992969 ( $n=25)$ measured in plasma from Hutterite children (all European ancestry). The asthma-associated risk allele at each SNP is highlighted in red (x axis). The number of subjects per group is shown below the genotype. Boxes

453 indicate the interquartile range, whiskers represent the $95 \%$ confidence intervals. Statistical significance 454 was determined using an additive linear model. 


\section{Discussion}

We described an integrated pipeline to fine-map and functionally annotate the

457 asthma-associated locus that includes the IL33 gene. We used the LD structure at this

458 locus across populations of different ethnicities combined with a Bayesian fine-mapping

459 tool to define a critical $20 \mathrm{~kb}$ genomic interval containing candidate causal SNPs for the

460 asthma association. Epigenetic signatures further reduced this region to $5 \mathrm{~kb}$, which we

461 demonstrated to have a crucial role in the development of chromatin loops creating

462 contacts between IL33 promoters and regulatory elements within the critical interval to

463 control IL33 expression. Associations between rs1888909 (T) allele copies and

464 increased IL33 mRNA and IL-33 protein levels further suggests that this regulatory

465 variant mediates the development of asthma among individuals carrying this risk allele.

466 The $5 \mathrm{~kb}$ region that we delineated and characterized in this study defies the

467 standard definition of regulatory elements. A loss of function assay showed that this

468 region is necessary for IL33 expression in vivo, suggestive of an enhancer function.

469 However, we failed to detect enhancer activity in reporter assays in cells where the

470 region displays chromatin markers associated with enhancers. Moreover, ENCODE

471 profiled the binding of hundreds of transcription factors across multiple cell types and

472 reported no binding of transcription activators, transcriptional co-factors, RNA Polll or

473 other factors usually associated with enhancers within this $5 \mathrm{~kb}$ region. Rather, the

474 region is bound by CTCF and cohesin in most cell lines assayed by ENCODE. We

475 showed that this region possesses enhancer blocking activity in vivo and in vitro,

476 reminiscent of insulator activities. Nevertheless, the region does not appear to behave

477 as a classically described insulator, as we fail to see a change in chromatin topology

478 and long-range interactions in the locus secondary to the deletion of the $5 \mathrm{~kb}$ region in 
479 the human BAC, which are typically seen when insulators are deleted ${ }^{33}$. While we 480 cannot ascribe a well-defined category for this regulatory element, we were able to

481 dissect its functional properties, to fine-map a variant that is likely causal to the 482 association with asthma and to identify the molecular effector binding to this regulatory 483 variant.

$484 \quad$ CTCF plays a critical role in chromatin loop formation and participates in 485 demarcation of Topological Association Domains (TADs). Loop formation within TADs 486 facilitates contacts between specific genes and enhancers, allowing for appropriate 487 temporal and tissue-specific expression ${ }^{34}$. We observed that the $5 \mathrm{~kb}$ risk haplotype 488 carrying the asthma-associated allele possessed reduced enhancer blocking function in 489 both in vitro and in vivo assays, and identified a smaller $1 \mathrm{~kb}$ fragment which maintained 490 enhancer blocking activity. Interestingly, the loss of a 400bp region within this $1 \mathrm{~kb}$ 491 fragment, harboring 2 asthma-associated SNPs, led to a significant loss of function, 492 demonstrating a requirement for factors binding in that region for its proper regulatory 493 activity.

494 We identified OCT-1 as a transcription factor that binds differentially to the risk 495 allele of rs 1888909 , thus implicating OCT-1 in the function(s) of this $5 \mathrm{~kb}$ region. OCT-1 496 can regulate gene expression both positively and negatively. It has been shown to bind 497 enhancers and regulatory regions upstream of multiple cytokines, including IL-3 ${ }^{35}$, IL$49812 \mathrm{p} 40^{36}, \mathrm{IL}-13^{37}, \mathrm{IL}-4^{38}$ and IL-1 $17^{39}$. The mechanisms by which OCT-1 functions are 499 strikingly diverse, and there are numerous studies reporting the coordinated activity of 500 CTCF and OCT-1. For example, at the IL17 gene cluster on chromosome 3, OCT-1 and 501 CTCF facilitate long-range associations with the IL-2 locus in naïve T cells. In a parallel 
502 fashion, it is possible that OCT-1 coordinates with CTCF to direct IL33 expression at the 503 locus through creation of appropriate chromatin interactions between enhancers and the

504 IL33 promoter. The distinct binding complexes formed on the risk and non-risk alleles at 505 the rs1888909 genomic region may be responsible for alterations in these interactions, 506 which in turn may lead to altered IL33 expression levels.

$507 \quad$ While our data support a role of rs1888909 in the regulation of IL33 expression 508 and its primary role with asthma risk, our fine mapping results identified two other 509 potential causative sets of SNPs associated with asthma at the IL33 locus, upstream 510 and downstream to the region that we studied (Fig1b). This suggests that other variants

511 in this locus may have independent effects that alter the function of other regulatory

512 elements and potentially control expression of IL33 or other genes. Future studies will

513 be needed to further dissect the genetic architecture at this complex locus and address 514 this hypothesis.

515 In summary, we identified a small $5 \mathrm{~kb}$ noncoding interval which is integral to 516 IL33 expression in several cell types. We propose a model in which CTCF mediates 517 interaction of the $5 \mathrm{~kb}$ region to the IL33 promoters through the formation of chromatin 518 loops. The interplay between the distinct regulatory elements in the locus promotes 519 spatial and temporal-specific regulation which is affected by OCT-1 binding to 520 rs1888909. These results together with the increased IL33 expression observed in 521 humans with the risk allele offer a plausible mechanistic explanation for the association 522 of these variants and asthma risk. A deeper characterization of these mechanisms will 523 not only enhance our understanding of IL33 regulation but may also open new 
524 strategies in directing future studies, as well as other common diseases in which 525 dysregulation of IL-33 plays a role. 


\section{Materials and Methods.}

\section{Experimental animals}

528 Generation of $\mathrm{hIL} 33^{\mathrm{Crm}} \mathrm{BAC}$ and $\mathrm{h} / L 33^{\mathrm{Crm}} 5 \mathrm{Kdel}$ transgenic mice was performed by the

529 University of Chicago Transgenic Core Facility. Modified DNA was diluted to a

530 concentration of $2 \mathrm{ng} / \mu \mathrm{l}$ and used for pronuclear injections of CD1 embryos in

531 accordance with standard protocols approved by the University of Chicago.

\section{Red/ET BAC modification}

534 BAC RP11725F15, obtained from the BACPAC Resource Center (Oakland, CA), was

535 modified in vitro using the RED/ET recombination kit (Gene Bridges, Heidelberg

536 Germany) according to the manufacturer's instructions.

537 Crimson- kanamycin cassette was PCR amplified from the pE2-Crimson-N1 vector. The

538 reporter cassette was inserted in frame to the ATG of the human IL33 gene, to replace

539 the second exon of the gene, while maintaining fully intact boundaries and flanking

540 regions. Primers containing 50-bp BAC-homology arms used for generation of the

541 recombination cassette were as follows: IL33_CrimsonKanF:

542 TTGAGACAAATGAACTAATATTATATTTTAATCCAACAGAATACTGAAAAATGGATAG

543 CACTGAGAACGTCA; IL33_CrimsonKanR,

544 GCGTAAAACATTCAGAGATAACTTAAGTCCTTACTTCCCAGCTTGAAACATCAGAAG

545 AACTCGTCAAGAAGG. Successful recombinants were screened for homologous

546 insertion using the following external/internal primers IL33F-Crm5',

547 AGCCACAGTTGTTTCCGTTT; IL33R-Crm5', TTGAGGTAGTCGGGGATGTC and IL548 33F-Crm3', ATCGCCTTCTATCGCCTTCT; IL33R, Crm3', 
550 The asthma-associated $5 \mathrm{~kb}$ interval (chr9: 6,194,500-6,199,500; hg19) was deleted

551 using RED/ET recombination kit (Gene Bridges). The $5 \mathrm{~kb}$ region of interest was

552 replaced by the ampicillin gene using the following primers containing $50 \mathrm{pb}$ homology

553 arms flanking the BAC region to be deleted. IL33InsDEL_F,

554 GCACACCTGTAAGTCTCTGCATTTTGCCACTTATACAACTTCATCTTTGAGTGGCAC

555 TTTTCGGGGAAATG; IL33InsDEL_R,

556 AAACTTACATCAAATAAAATCTCAACACAGAATTCATACATGTCAACATACTCGAGG

557 CTAGCTCTAGAAGTC. All correctly modified BACs were verified by fingerprinting and

558 sequencing. BAC DNA was extracted using the Nucleobond PC20 kit (Macherey-Nagel)

559 and diluted for pronuclear injection. BAC copy number was determined as previously

560 described ${ }^{40}$

562 Quantitative RT-PCR

563 RNA from flash frozen tissues were extracted from control and insulator deleted mice

564 using TRI-reagent (Sigma). cDNA was generated using Super Script II (Thermo). IL33

565 primers (5'GCCAAGCTGCAAGTGACCAA 3'; 5'GCCTTGGAGCCGTAGAAGAA 3') and

566 HPRT housekeeping gene primes were used to quantify IL33 mRNA expression levels.

567 RNA samples with no reverse transcriptase added were used to test for genomic 568 contamination. 


\section{Tissue preparation, Immunofluorescence Staining, and Microscopy}

573 Mouse lymph nodes were embedded into blocks with Optimal Cutting Temperature

574 compound (OCT 4583) and stored at $-80^{\circ} \mathrm{C}$. Frozen tissues were sliced into sections

$5755 \mu \mathrm{m}$ thick and dried onto slides overnight. Sections were fixed, permeabilized,

576 quenched and blocked. Tissues were immunostained with the primary antibodies rat

577 anti-mouse CD31:Biotin (clone 390, Biolegend, San Diego, CA, USA) and Living Colors

578 DsRed Polyclonal Antibody (rabbit anti-E2-Crimson, Clontech [now Takara Bio USA],

579 Mountain View, CA, USA). Sections were washed and stained with the secondary

580 antibodies Streptavidin:Alexa Fluor 488 (Biolegend, San Diego, CA, USA) and Goat

581 anti-Rabbit IgG:Alexa Fluor 633 (Life Technologies, Thermo Fisher, Waltham, MA,

582 USA), along with the nucleic acid stain Hoechst 33342 (Life Technologies, Thermo

583 Fisher, Waltham, MA, USA). Coverslips were set with ProLong Diamond Antifade

584 Mountant (Life Technologies, ThermoFisher, Waltham, MA, USA). Imaging was

585 performed at the University of Chicago Integrated Light Microscopy Facility. Images

586 were captured with a Leica TCS SP2 laser scanning confocal microscope (Leica

587 Microsystems, Inc., Buffalo Grove, IL, USA) using a 63x/1.4 UV oil immersion objective

588 and LAS_AF acquisition software (Leica Microsystems, Inc., Buffalo Grove, IL, USA).

589 Further processing was completed using ImageJ software (National Institutes of Health,

590 Bethesda, MD, USA).

591

\section{Circularized Chromosome Conformation Capture sequencing (4C-seq)}

593 4C-seq assays were performed as previously reported ${ }^{9} .20$ million bone marrow derived

594 dendritic cells were obtained from control and insulator deleted mice. Immediately before 
595 4C-seq library preparation, cells were cross-linked with $1 \%$ fresh formaldehyde for 15 596 minutes and treated with lysis buffer (10 mM Tris- $\mathrm{HCl} \mathrm{pH} 8,10 \mathrm{mM} \mathrm{NaCl}, 0.3 \%$ IGEPAL 597 CA-630 (Sigma-Aldrich), 1X complete protease inhibitor cocktail (Roche). Nuclei were 598 digested with Csp6-I endonuclease (Life Technologies, ThermoFisher, Waltham, MA) 599 and ligated with T4 DNA ligase (Promega, Madison, WI) for 12 hours at $4^{\circ} \mathrm{C}$, followed by 600 reverse crosslinking at $65^{\circ} \mathrm{C}$ for 12 hours with proteinase K. Subsequently, Nlalll 601 endonuclease (New England Biolabs) was used in a second round of digestion, and the 602 DNA was ligated again. Specific primers were designed near the $5 \mathrm{~kb}$ region of interest. 603 Viewpoint fragment ends (fragends) coordinate was chr9:6,186,164-6,186,468. Primers 604 used for 4C-Seq experiments are as follows: 4CmIL33IL33Enh_nonread, 5' 605 CAAGCAGAAGACGGCATACGAACAACTTCACTCAGAGGCATG 606 4CmIL33IL33Enh_Read 5'

607 AATGATACGGCGACCACCGAACACTCTTTCCCTACACGACGCTCTTCCGATCTGAG 608 ATGGCGCCACTGTAC 3'

\section{Construct preparation}

611 PCR fragments of genomic DNA from a risk and non-risk cell line were cloned into the 612 pDONR vector (Life Technologies, ThermoFisher, Waltham, MA) and sequenced by the 613 University of Chicago DNA Sequencing and Genotyping facility. DNA was subcloned 614 into the enhancer barrier vector ${ }^{28}$ (gift from Dr. Laura Elnitski, NHGRI) or pGL4.23 for 615 enhancer assays (Promega, Madison, WI). Constructs were prepared using the Plasmid 616 MidiPrep Kit (Qiagen, Hilden, Germany) and re-sequenced to confirm genotype. 
619 K562 cells were transfected using TransIT-2020 Transfection Reagent (Mirus Bio,

620 Madison, WI). Briefly, $10^{5}$ cells per well were plated 24 hours prior to transfection. The

621 transfection mix included $0.5 \mu \mathrm{g}$ of barrier or enhancer plasmid, 10ng hprl normalization

622 control plasmid, and $1.5 \mu$ l transfection reagent. TeloHaecs were transfected using

623 JetPRIME transfection reagent (Polyplus). $2.10^{4}$ cells per well in 24 well plates were

624 transfected 3 days later with 250ng of test plasmid DNA and $25 \mathrm{ng}$ of hprl normalization

625 control plasmid. After 48 hours cells were harvested and assayed for luciferase with a

$62620 / 20^{\text {n }}$ luminometer (Turner Biosystems, now Fisher Scientific, Hampton, NH) using the

627 Dual Luciferase Reporter Assay System (Promega, Madison, WI). Firefly luciferase was

628 normalized to the Renilla luciferase. A minimum of three independent transfections

629 using three different DNA preparations were assayed.

\section{Zebrafish transgenesis}

632 The Tol2 vector contains a strong midbrain enhancer, a Gateway entry site and the

633 cardiac actin promoter controlling the expression of EGFP, and was developed to

634 screen for insulator activity ${ }^{29}$. Each candidate sequence was recombined between the

635 midbrain enhancer and the cardiac actin promoter. As a reference, the empty backbone

636 was used (INS-zero). One cell-stage embryos were injected with 3-5 nL of a solution

637 containing $25 \mathrm{nM}$ of each construct plus $25 \mathrm{nM}$ of Tol2 mRNA. Embryos where then

638 incubated at $28^{\circ} \mathrm{C}$ and EGFP expression was evaluated $24 \mathrm{hpf}$. The midbrain/somites

639 EGFP intensity ratio was quantified using ImageJ freeware and was directly proportional

640 to the enhancer-blocking capacity. As a positive control, the chicken beta-globin 
641 insulator 5HS4 was used. Each experiment was repeated independently and double-

642 blinded to the operators.

\section{EMSA}

645 Nuclear extracts were prepared from K562 cells using NE-PER Nuclear and 646 Cytoplasmic Extraction Kit (Thermo Fisher, Waltham, MA) supplemented with HALT

647 Protease Inhibitor Cocktail and PMSF (Thermo Fisher, Waltham, MA). Protein

648 concentrations were determined with the BCA kit (Thermo Fisher, Waltham,MA).

649 Oligonucleotides to be used as probes were synthesized with a 5'IRDye 700

650 modification (IDT). Binding reactions were performed using the Odyssey EMSA kit (LI-

651 COR Biosciences, Lincoln, NE) and contained $5 \mu$ g nuclear extract, 2.5nM labeled

652 probe, and 100x excess unlabeled oligonucleotide when noted. For supershift assays,

$6532 \mu \mathrm{g}$ Oct-1 antibody (Santa Cruz Biotechnology, Dallas, TX, sc-232) was added and

654 incubated for another 20 minutes. Reaction mixtures were run on a $4 \%$ nondenaturing 655 polyacrylamide gel and analyzed with the LI-COR Odyssey Imaging System.

657 Oligonucleotide/Probe sequences:

658 rs10975479G:rs1888909T:TCTGATGCAGAACAGCAATGTGTTTTCCAIGTGCACTTGGTC 659 rs10975479G:rs1888909C:CCTGATGCAGAACAGCAATGTGTTTTCCACGGTGCACTTGGTC 660 rs10975479A:rs1888909T:TCTGATGCAGAACA_CAATGTGTTTTCCA_GTGCACTTGGTC 661 rs10975479A:rs1888909C:CCTGATGCAGAACA_ACAATGTGTTTTCCACGGTGCACTTGGTC 662 Consensus OCT-1 TGTCGAATGCAAATCACTAGAA

663 Mutant OCT-1 TGTCGAATGCAAGCCACTAGAA. 


\section{Mass Spectrophotometry and protein identification}

665 The sections to be analyzed from the EMSA gel were excised, washed and destained 666 using $100 \mathrm{mM} \mathrm{NH} 4 \mathrm{HCO} 3$ in $50 \%$ acetonitrile (ACN). Sections then underwent reduction, 667 alkylation, and trypsinization. Peptides were extracted with 5\% formic acid, followed by $66875 \%$ ACN in formic acid, and cleaned up with C-18 spin columns (Pierce). The samples

669 were analyzed via electrospray tandem spectrometry (LC-MS/MS) on a Thermo Q-

670 Exactive Orbitrap mass spectrometer at the Proteomics Core at Mayo Clinic, Rochester,

671 Minnesota. Tandem mass spectra were extracted and then analyzed by Mascot and

672 X!Tandem algorithms. Scaffold v4.8.4 (Proteome Software Inc., Portland,OR) was used

673 to validate the protein identifications. Peptide identifications were accepted if they could

674 be established at greater than $98 \%$ probability to achieve an FDR less than $1.0 \%$.

\section{Candidate Transcription factor analysis}

677 Panther pathway analysis database ${ }^{41}$ was used to filter for DNA-binding proteins.

678 Jaspar (http://jaspar.genereg.net) and Alggen-promo (http://alggen.Isi.upc.es)

679 databases were used to screen these factors for known DNA binding sites. We

680 downloaded binding data for random octamers from UniProbe

681 (http://thebrain.bwh.harvard.edu/pbms/webworks pub dev/downloads.php) and

682 identified all transcription factors that bound at least one octamer containing a risk or

683 non-risk allele.

684

685 


\section{Chromatin Immunoprecipitation}

687 ChIP assays were performed using the Millipore ChIP assay kit and protocol. $2 \times 10^{6}$

$688 \mathrm{H} 292$ cells (heterozygous for the risk allele) were fixed with $1 \%$ formaldehyde.

689 Chromatin was sonicated using a diagenode BioRuptor. Lysates were incubated 690 overnight with 5ug of Oct-1 (Santa Cruz, sc-232x) or an IgG control (Santa Cruz, sc-

691 2027). Following the washes, elution of chromatin complexes and reversal of crosslinks,

692 DNA was recovered using Qiagen pcr purification kit. Input DNA was also processed.

693 QPCR was performed using the primers: F: 5'GCCTCTGGTCTCAGTGGATA3' and

694 R: 5'CTGCTCATAGGAGACACAGTAAAG3'.

696 Gene expression and genotype studies

697 RNA-seq data from airway epithelial cells were available from two sources. The first

698 was from bronchial epithelial cells sampled from adults (44 European American, 48

699 African American), with and without asthma (76 cases, 60 controls), who participated in

700 a study of asthma in Chicago. Procedures for bronchoscopy, cell and RNA processing,

701 and genotyping have been previously described ${ }^{42}$. For this study, normalized gene

702 expression counts were adjusted for age, sex, current smoking status, sequencing pool,

703 the first three ancestry PCs. Linear regression considering additive genotype effects on

704 gene expression was performed using limma in $\mathrm{R}$ (v3.3.3). $P$-values were adjusted for 3

705 tests; $P<0.016$ was considered significant. All subjects provided written informed

706 consent; these studies were approved by University Chicago Institutional Review Board.

707 The second source was from nasal epithelial cells sampled from 246 African American

708 children (125 cases, 121 controls) from a birth cohort of children at high risk for 
709 asthma ${ }^{43}$. Procedures for nasal brushing and cell processing followed standard

710 procedures ${ }^{44}$. Genotypes were determined using Illumina Multi-Ethnic Genotyping Array

711 (MEGA), and processed using standard $\mathrm{QC}^{42}$. To test for association between

712 genotypes for three SNPs and IL33 transcript levels, we used an additive effects linear

713 model, including as covariates sex, study site, batch id, epithelial cell proportion and 12

714 latent factors in the epithelial cell studies. Latent factors were included to correct for

715 unwanted variation ${ }^{45}$. $P$-values were adjusted for 3 tests; $P<0.016$ was considered

716 significant. Parents of all children provided written informed consent, and children

717 provided written assent, for genetic studies; these studies were approved by University

718 Chicago Institutional Review Board.

$720 \quad$ IL-33 cytokine and genotype studies

721 Blood for cytokine studies was drawn from 30 Hutterite children during trips to South

722 Dakota, as previously described ${ }^{46}$. Written consent was obtained from the parents and

723 written assent was obtained from the children. The study was approved by the

724 institutional review boards at the University of Chicago. Briefly, The Milliplex Map

725 Human TH17 Magnetic Bead Panel (Millipore, Burlington, MA) was used to measure IL-

72633 levels in thawed supernatants at the University of Chicago Immunology Core facility

727 using standard protocols, as previously described. ${ }^{47}$

728 Associations between genotypes at rs1888909, rs992969 and rs10975479 and

729 normalized IL-33 cytokine abundance were tested using a linear model, assuming

730 additive effects. Prior to analysis, voom-transformed gene expression counts were

731 adjusted for age, sex, current smoking status, sequencing pool and the first three 
732 ancestry PCs using the function removeBatchEffect from the R package limma ${ }^{48}$. Linear

733 regression between the genotypes and IL-33 levels was performed with the FastQTL

734 software package ${ }^{49}$. One subject had poor IL-33 cytokine data and was removed. Of the

735 remaining subjects, 27 subjects for rs 10975479 and rs 1888909 and 25 subjects for

736 rs992969 had good quality genotype calls and were retained. Linear regression

737 between the genotypes and IL-33 cytokine levels was performed using limma, adjusting

738 for sex and age. Genotypes were obtained using PRIMAL ${ }^{50}$, an in-house pedigree-

739 based imputation tool that imputes variants from whole genome sequences from 98

740 Hutterite individuals to $>1600$ individuals who were genotyped with an Affymetrix

741 genotyping array.

742 


\section{Acknowledgements}

744 We thank Don Wolfgeher, from the University of Chicago's Proteomics Core Facility, for

745 assistance with sample preparation and analysis of the mass spectrophotometry; Dr.

746 Laura Elnitski (NHGRI/NIH, Bethesda,MD), for generously supplying the enhancer

747 barrier plasmids. Dr. Benjamin Glick (University of Chicago) for supplying the E2-

748 Crimson reporter vector and DiRienzo lab (University of Chicago) for the TeloHAECs

749 cells. This work was supported by NIH grants R01HL128075 (M.A.N.), R01HL119577

750 (M.A.N.), and R01HL 118758 (M.A.N. and A.I. S.).

\section{Disclosures}

754 The authors have no financial conflict of interest. 


\section{REFERENCES}

757 1. Polderman, T.J. et al. Meta-analysis of the heritability of human traits based on fifty years of twin studies. Nature genetics 47, 702-709 (2015).

2. Pividori, M., Schoettler, N., Nicolae, D.L., Ober, C. \& Im, H.K. Shared and distinct genetic risk

6. Savinko, T. et al. IL-33 and ST2 in atopic dermatitis: expression profiles and modulation by triggering factors. J Invest Dermatol 132, 1392-1400 (2012).

7. Kamekura, R. et al. The role of IL-33 and its receptor ST2 in human nasal epithelium with allergic rhinitis. Clin Exp Allergy 42, 218-228 (2012).

8. Kottyan, L.C. et al. Genome-wide association analysis of eosinophilic esophagitis provides insight into the tissue specificity of this allergic disease. Nature genetics 46, 895-900 (2014).

9. Smemo, S. et al. Obesity-associated variants within FTO form long-range functional connections with IRX3. Nature 507, 371-375 (2014).

10. van den Boogaard, M. et al. A common genetic variant within SCN10A modulates cardiac SCN5A expression. J Clin Invest 124, 1844-1852 (2014).

11. Rusu, V. et al. Type 2 Diabetes Variants Disrupt Function of SLC16A11 through Two Distinct Mechanisms. Cell 170, 199-212 e120 (2017).

12. Small, K.S. et al. Regulatory variants at KLF14 influence type 2 diabetes risk via a female-specific effect on adipocyte size and body composition. Nature genetics 50, 572-580 (2018).

13. Wasserman, N.F., Aneas, I. \& Nobrega, M.A. An 8q24 gene desert variant associated with prostate cancer risk confers differential in vivo activity to a MYC enhancer. Genome Res 20, 1191-1197 (2010).

14. Gallagher, P.G. et al. Mutation of a barrier insulator in the human ankyrin-1 gene is associated with hereditary spherocytosis. J Clin Invest 120, 4453-4465 (2010).

801 
802

15. Moffatt, M.F. et al. A large-scale, consortium-based genomewide association study of asthma. $N$ Engl J Med 363, 1211-1221 (2010).

16. Torgerson, D.G. et al. Meta-analysis of genome-wide association studies of asthma in ethnically diverse North American populations. Nature genetics 43, 887-892 (2011).

17. Bonnelykke, K. et al. A genome-wide association study identifies CDHR3 as a susceptibility locus for early childhood asthma with severe exacerbations. Nature genetics 46, 51-55 (2014).

18. Demenais, F. et al. Multiancestry association study identifies new asthma risk loci that colocalize with immune-cell enhancer marks. Nature genetics 50, 42-53 (2018).

19. Daya, M. et al. Association study in African-admixed populations across the Americas recapitulates asthma risk loci in non-African populations. Nature communications 10,880 (2019).

20. Ferreira, M.A.R. et al. Genetic Architectures of Childhood-and Adult-Onset Asthma Are Partly Distinct. Am J Hum Genet 104, 665-684 (2019).

21. Zhu, X. \& Stephens, M. Bayesian Large-Scale Multiple Regression with Summary Statistics from Genome-Wide Association Studies. Ann Appl Stat 11, 1561-1592 (2017).

22. Bycroft, C. et al. The UK Biobank resource with deep phenotyping and genomic data. Nature 562, 203-209 (2018).

23. Roadmap Epigenomics, C. et al. Integrative analysis of 111 reference human epigenomes. Nature 518, 317-330 (2015).

24. Consortium, E.P. A user's guide to the encyclopedia of DNA elements (ENCODE). PLoS Bio/ 9, e1001046 (2011).

25. Kim, S., Yu, N.K. \& Kaang, B.K. CTCF as a multifunctional protein in genome regulation and gene expression. Exp Mol Med 47, e166 (2015).

26. Chen, D. \& Lei, E.P. Function and regulation of chromatin insulators in dynamic genome organization. Curr Opin Cell Biol 58, 61-68 (2019).

27. Pichery, M. et al. Endogenous IL-33 Is Highly Expressed in Mouse Epithelial Barrier Tissues, Lymphoid Organs, Brain, Embryos, and Inflamed Tissues: In Situ Analysis Using a Novel II-33-LacZ Gene Trap Reporter Strain. J Immunol 188, 3488-3495 (2012).

28. Petrykowska, H.M., Vockley, C.M. \& Elnitski, L. Detection and characterization of silencers and enhancer-blockers in the greater CFTR locus. Genome Res 18, 1238-1246 (2008).

29. Bessa, J. et al. Zebrafish enhancer detection (ZED) vector: a new tool to facilitate transgenesis and the functional analysis of cis-regulatory regions in zebrafish. Dev Dyn 238, 2409-2417 (2009). 
30. Hume, M.A., Barrera, L.A., Gisselbrecht, S.S. \& Bulyk, M.L. UniPROBE, update 2015: new tools and content for the online database of protein-binding microarray data on protein-DNA interactions. Nucleic Acids Res 43, D117-122 (2015).

31. Li, X. et al. eQTL of bronchial epithelial cells and bronchial alveolar lavage deciphers GWASidentified asthma genes. Allergy (2015).

32. Stein, M.M. et al. Innate Immunity and Asthma Risk in Amish and Hutterite Farm Children. $N$ Engl J Med 375, 411-421 (2016).

33. Flavahan, W.A. et al. Altered chromosomal topology drives oncogenic programs in SDH-deficient GISTs. Nature 575, 229-233 (2019).

34. West, A.G., Gaszner, M. \& Felsenfeld, G. Insulators: many functions, many mechanisms. Genes Dev 16, 271-288 (2002).

35. Duncliffe, K.N., Bert, A.G., Vadas, M.A. \& Cockerill, P.N. A T cell-specific enhancer in the interleukin-3 locus is activated cooperatively by Oct and NFAT elements within a DNase Ihypersensitive site. Immunity 6, 175-185 (1997).

36. Zhou, L. et al. An inducible enhancer required for $1112 \mathrm{~b}$ promoter activity in an insulated chromatin environment. Mol Cell Biol 27, 2698-2712 (2007).

37. Kiesler, P., Shakya, A., Tantin, D. \& Vercelli, D. An allergy-associated polymorphism in a novel regulatory element enhances IL13 expression. Hum Mol Genet 18, 4513-4520 (2009).

38. Kim, K., Kim, N. \& Lee, G.R. Transcription Factors Oct-1 and GATA-3 Cooperatively Regulate Th2 Cytokine Gene Expression via the RHS5 within the Th2 Locus Control Region. PLoS One 11, e0148576 (2016).

39. Kim, L.K. et al. Oct-1 regulates IL-17 expression by directing interchromosomal associations in conjunction with CTCF in T cells. Mol Cell 54, 56-66 (2014).

40. Chandler, K.J. et al. Relevance of BAC transgene copy number in mice: transgene copy number variation across multiple transgenic lines and correlations with transgene integrity and expression. Mamm Genome 18, 693-708 (2007).

41. Mi, H., Muruganujan, A., Ebert, D., Huang, X. \& Thomas, P.D. PANTHER version 14: more genomes, a new PANTHER GO-slim and improvements in enrichment analysis tools. Nucleic Acids Res 47, D419-D426 (2019).

896

42. Nicodemus-Johnson, J. et al. DNA methylation in lung cells is associated with asthma endotypes and genetic risk. JCl Insight 1, e90151 (2016).

43. Gern, J.E. et al. The Urban Environment and Childhood Asthma (URECA) birth cohort study: design, methods, and study population. BMC Pulm Med 9, 17 (2009). 
897

903

904

905

906

907

908

909

910
44. Poole, A. et al. Dissecting childhood asthma with nasal transcriptomics distinguishes subphenotypes of disease. J Allergy Clin Immunol 133, 670-678 e612 (2014).

45. McKennan, C. \& Nicolae, D.L. Accounting for unobserved covariates with varying degree of estimability in high dimensional experimental data. Biometrika 106, 823-840 (2019).

46. Stein, M.M. et al. Innate Immunity and Asthma Risk in Amish and Hutterite Farm Children. N Engl J Med 375, 411-421 (2016).

47. Stein, M.M., Hrusch, C.L., Sperling, A.I. \& Ober, C. Effects of an FcgammaRIIA polymorphism on leukocyte gene expression and cytokine responses to anti-CD3 and anti-CD28 antibodies. Genes Immun (2018).

48. Ritchie, M.E. et al. limma powers differential expression analyses for RNA-sequencing and microarray studies. Nucleic Acids Res 43, e47 (2015).

49. Ongen, H., Buil, A., Brown, A.A., Dermitzakis, E.T. \& Delaneau, O. Fast and efficient QTL mapper for thousands of molecular phenotypes. Bioinformatics 32, 1479-1485 (2016).

50. Livne, O.E. et al. PRIMAL: Fast and Accurate Pedigree-based Imputation from Sequence Data in a Founder Population. PLoS Comput Biol 11, e1004139 (2015). 
Figures

a

b

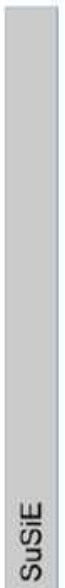

C

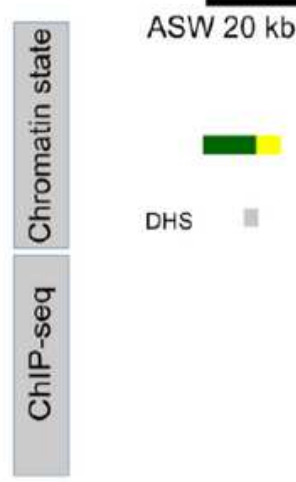

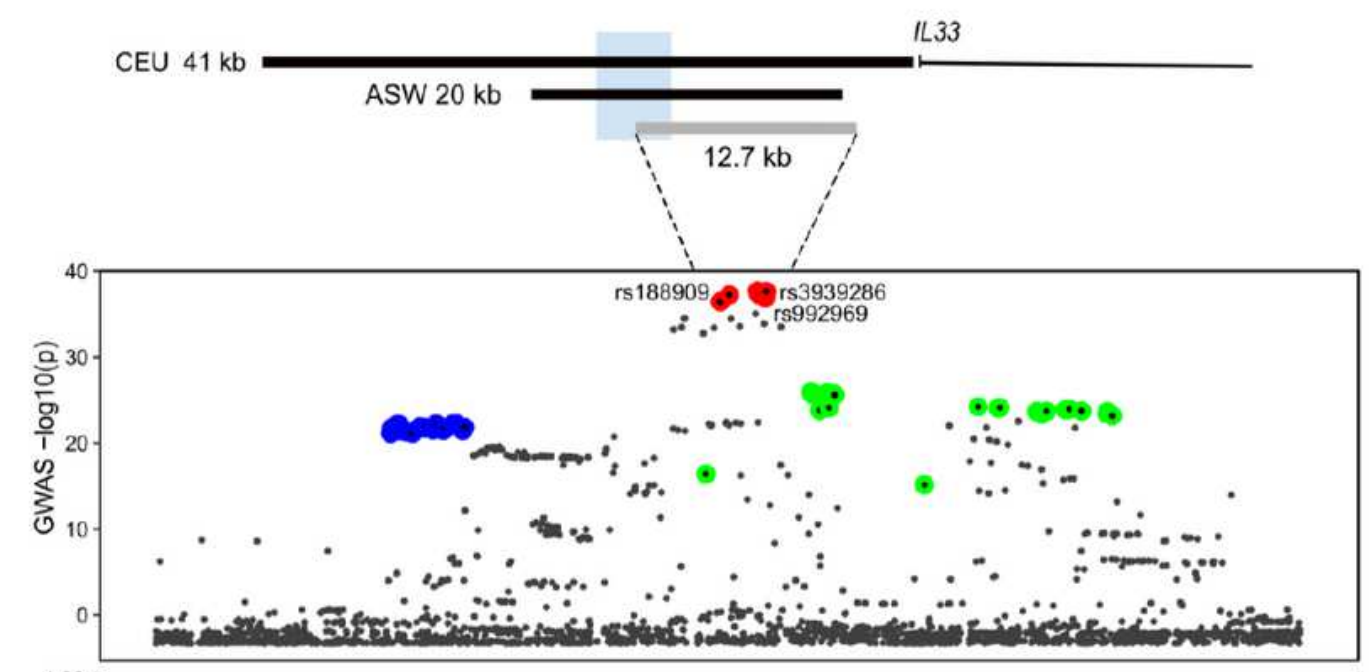
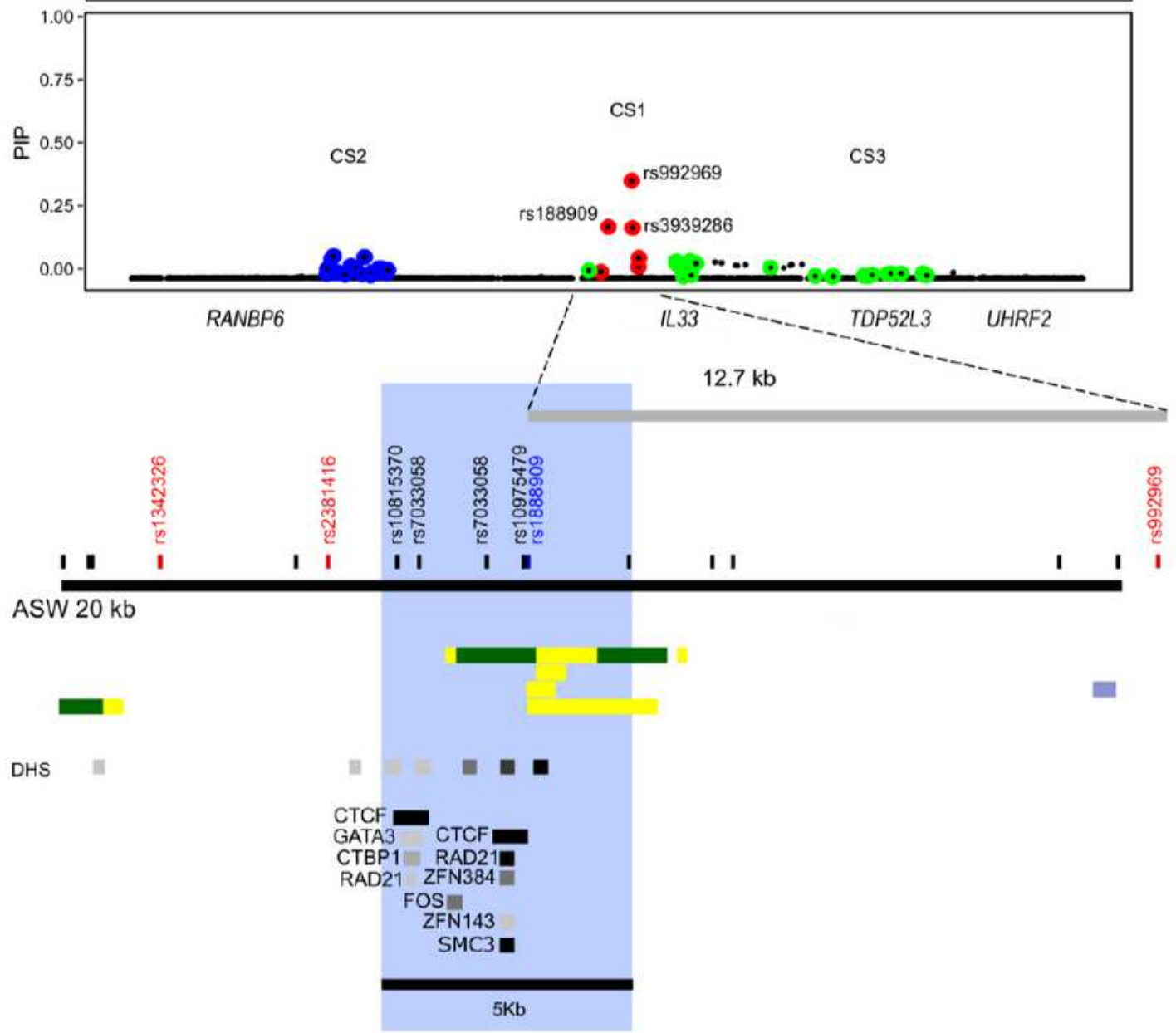

Figure 1

Epigenetic characterization of the asthma-associated critical region in the IL33 locus. a Schematic organization of the IL33 gene and the asthma-associated region (black bars) of European ancestry (CEU 41 kb, chr9: 6,172,380-6,213,468; hg19) and African ancestry (ASW 20 kb, chr9: 6,188,124-6,209,099; 
hg19) positioned upstream of exon 1. b Significance of SNP association in the GWAS2 (top) and fine mapping results (bottom) for each variant at the IL33 locus (bottom). Colors indicate each credible set (CS) identified. CS1: red, CS2 blue; CS3 green. CS1 (rs992969, rs1888909, rs3939286) defines a region of $12.7 \mathrm{~kb}$ (chr9: 6,197,392 6,210,099; hg19). c Position of the lead GWAS SNPs (in red) and additional SNPs in high LD $(r 2 \geq 0.8)$ with the lead SNPs (in black) within the ASW $20 \mathrm{~kb}$ LD region. The lead SNP rs1888909 in African ancestry is shown in blue. Chromatin states from Roadmap Epigenomics Project showing regions with potential regulatory activity. Yellow: active enhancer; green: transcribed sequence; blue: heterochromatin. DNase hypersensitive (DHS) sites indicating open chromatin regions are shown. Tissues (from the top): E096 Lung primary HMM; E095 Left ventricle primary HMM; E116 GM128781 Lymphoblastoid cell primary HMM; E122 HUVEC Umbilical Vein Endothelial Primary Cells Primary HMM d ChIP-seq data from ENCODE-3 cell lines (338 factors; 130 cell types) showing co-binding of CTCF, RAD2, ZFNs and SMC-3 at the $5 \mathrm{~kb}$ interval (blue shaded region; chr9: 6,194,500-6,199,500; hg19). 
a

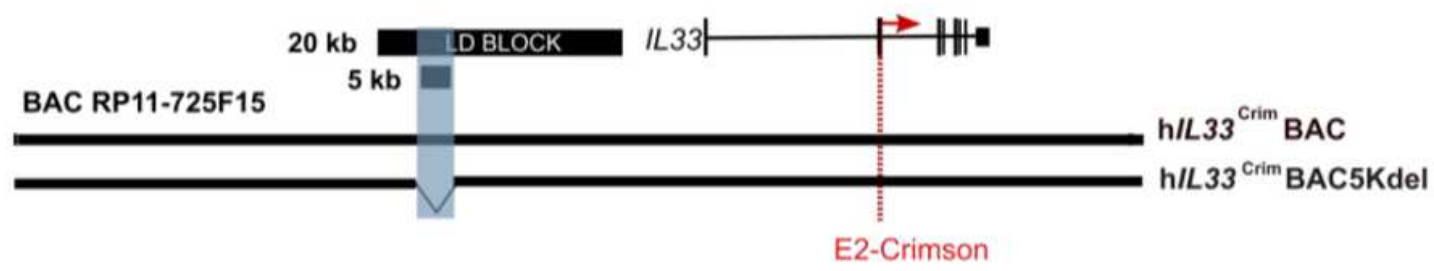

b
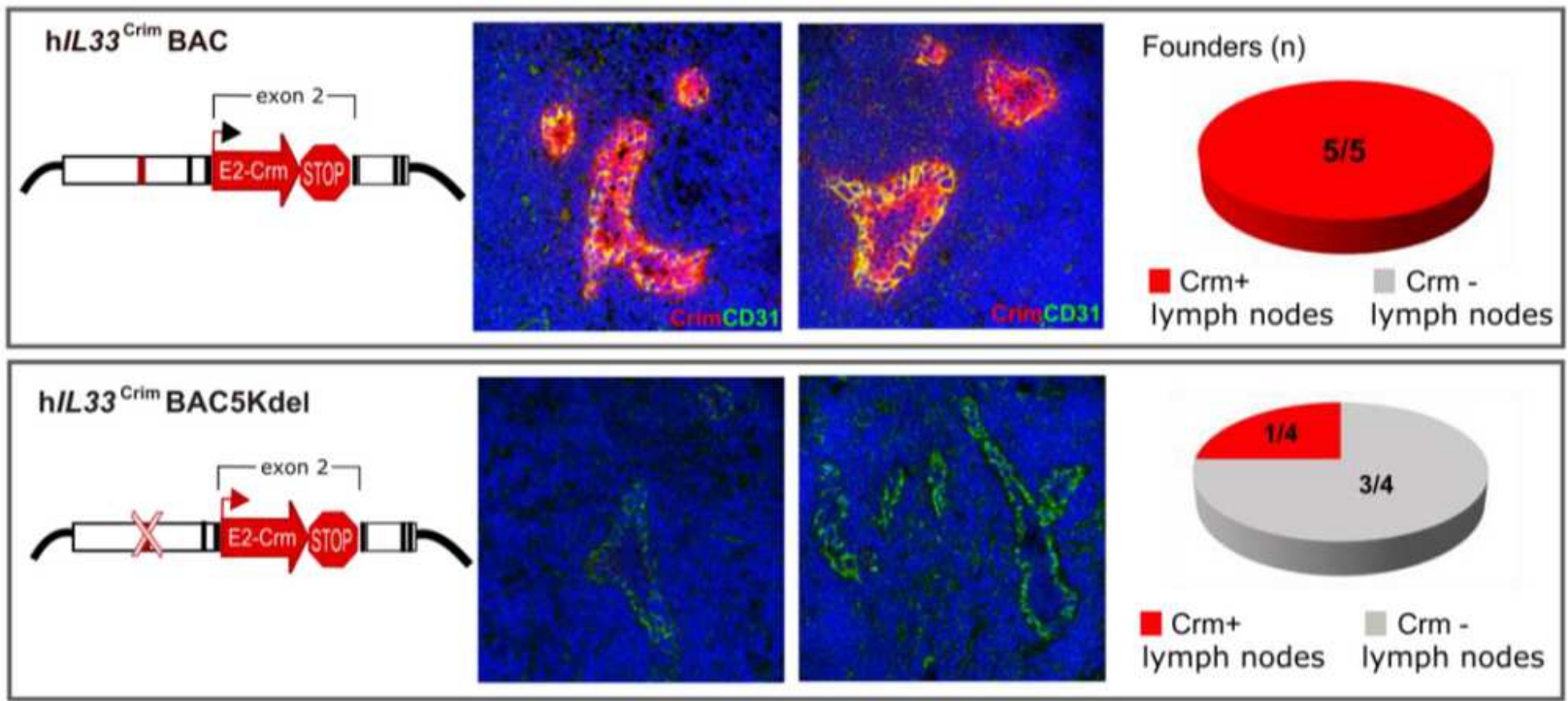

c

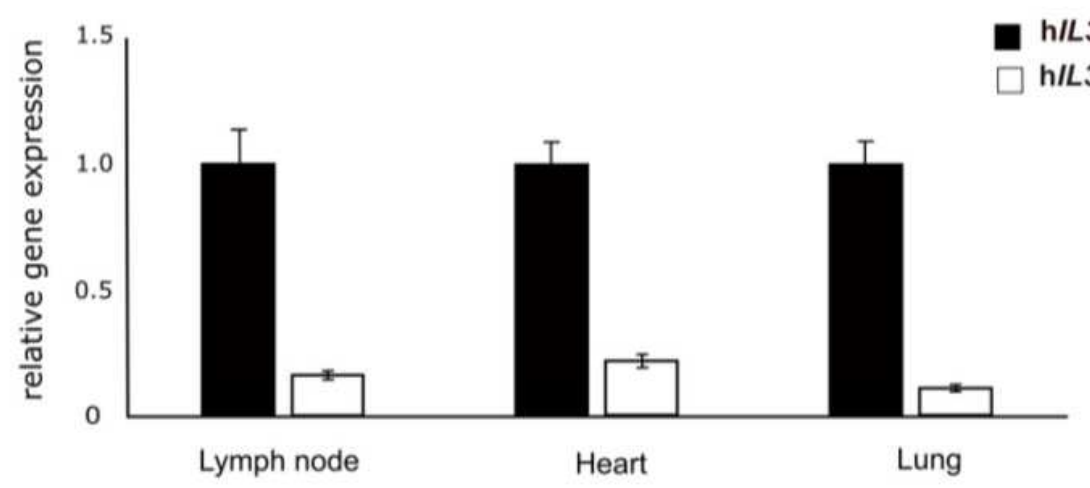

Figure 2

The IL33-containing BAC in transgenic mice encodes human-specific regulatory patterns and demonstrates the importance of the $5 \mathrm{~kb}$ noncoding segment for proper IL33 expression. a Schematic of human BAC clone RP11-725F15 (166 kb) spanning the entire coding region of IL33 and its upstream region including the $20 \mathrm{~kb}$ asthma-associated interval and the $5 \mathrm{~kb}$ region of interest shaded in blue (black bars). To produce a human IL33 reporter strain, a cassette containing E2-Crimson with a stop sequence was inserted into exon 2, in frame with the IL33 translational start site (red dotted line). Transgenic mice were generated with either the full BAC (hIL33CrimBAC) or a BAC containing a deletion of the $5 \mathrm{~kb}$ interval within the LD block (hIL33Crim BAC5Kdel). b Immunofluorescence staining of mouse peripheral lymph node sections of E2-Crimson in hIL33Crim BAC mice (upper panels) or hIL33Crim 
BAC5Kdel (lower panels). Representative founder BAC transgenic lines are shown. Sections were stained with anti-E2-Crimson (red) and the mouse endothelial cell marker CD31 (green). Hoechst staining for nuclei is in blue. c qPCR analysis of E2-Crimson mRNA obtained from lymph node (LN), heart and lung from both BAC strains is shown

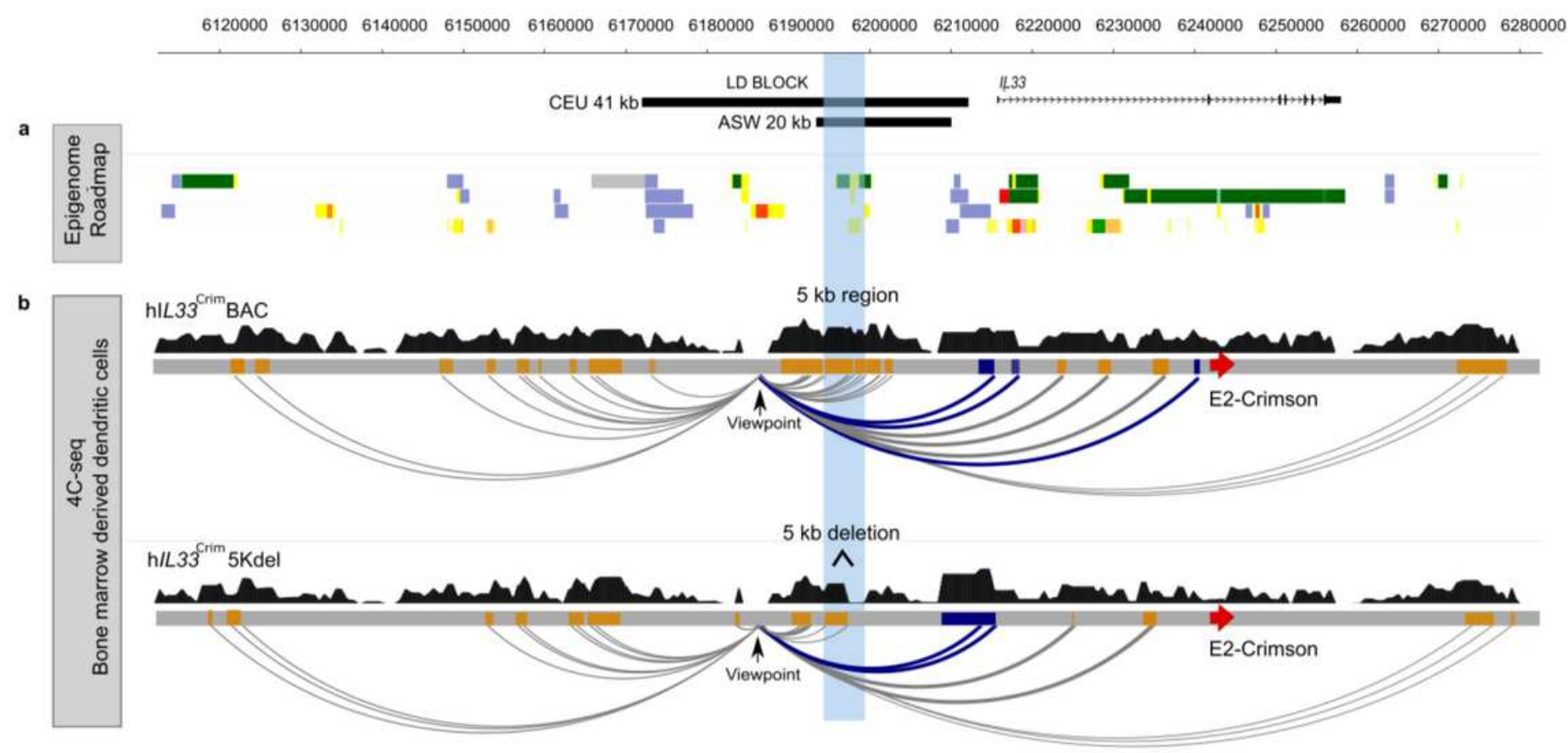

Figure 3

Interaction maps show looping of the asthma-associated region to the IL33 promoter. a Roadmap Epigenome data from heart (E095 Left ventricle primary HMM), lung (E096 Lung primary HMM), LCL (E116 GM128781 Lymphoblastoid cell primary HMM) and endothelial cells (E122 HUVEC Umbilical Vein Endothelial Primary Cells Primary HM). Chromatin state assignments are indicated as: transcribed, green; active enhancer, yellow; active promoter, red; repressed, gray; heterochromatin, blue. b 4C-seq in bone marrow derived dendritic cells obtained from mice containing the BAC (hIL33CrimBAC, top) and a deletion of the $5 \mathrm{~kb}$ interval within the LD block (hIL33Crim BAC5Kdel, bottom). Gray bars correspond to a schematic representation of the human BAC showing the E2-Crimson reporter insertion site on exon 2 of the IL33 gene (red arrow) and the asthma associated $5 \mathrm{~kb}$ region (blue shaded box). In both experiments, reads were mapped to the coordinates corresponding to the human BAC (chr9: 6,112,733-6,279,294; hg19) and peaks shown in dark blue and orange represent the promoter and distal elements, respectively. Arcs depict interactions from the viewpoint located upstream the $5 \mathrm{~kb}$ region. Interactions between the LD region, near to the $5 \mathrm{~kb}$ region (viewpoint), and the IL33 promoters are noted in blue 


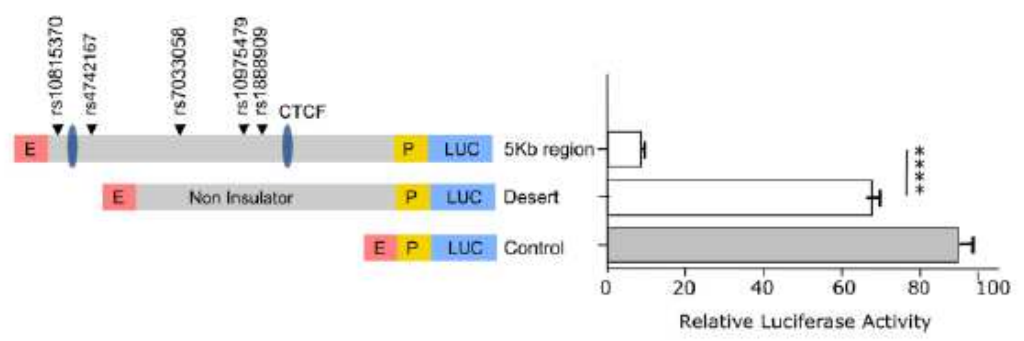

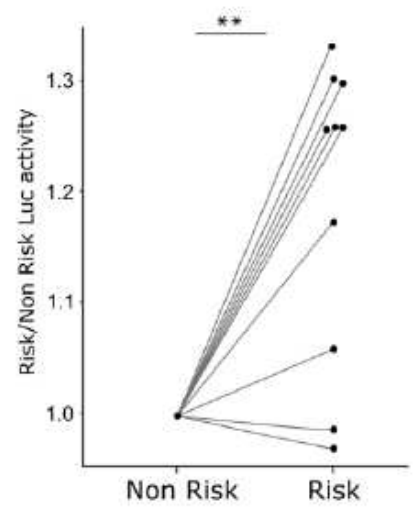

d

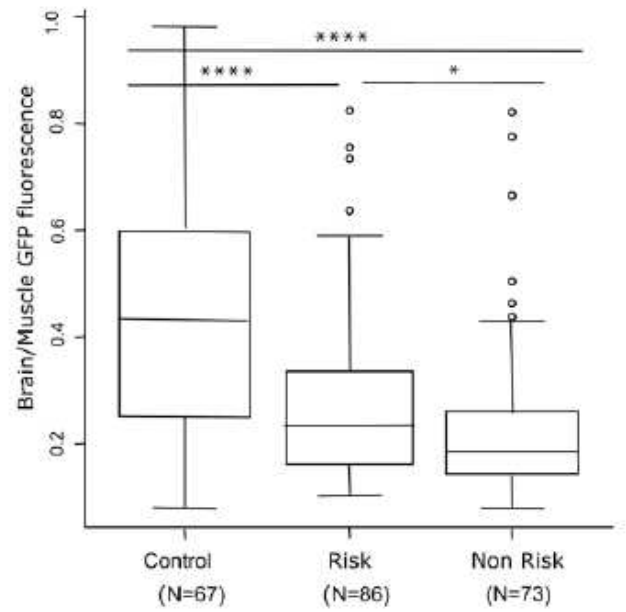

\section{d}

$\nabla$

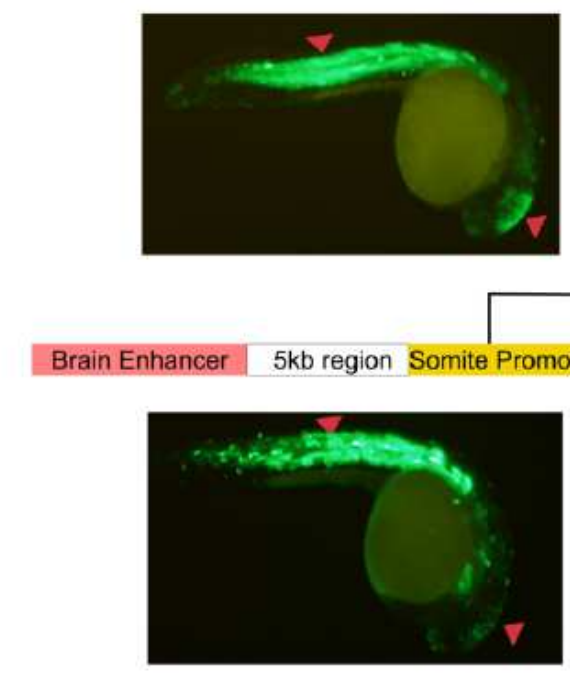

Brain Enhancer $5 \mathrm{~kb}$ region Somite Promoter GFP

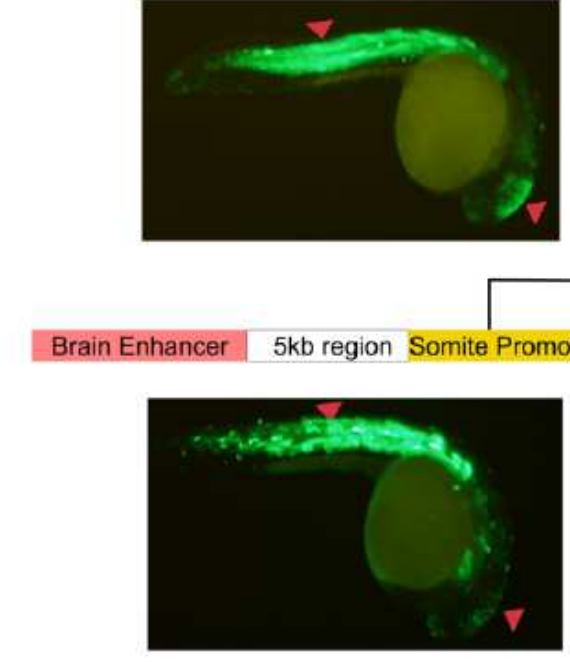

e

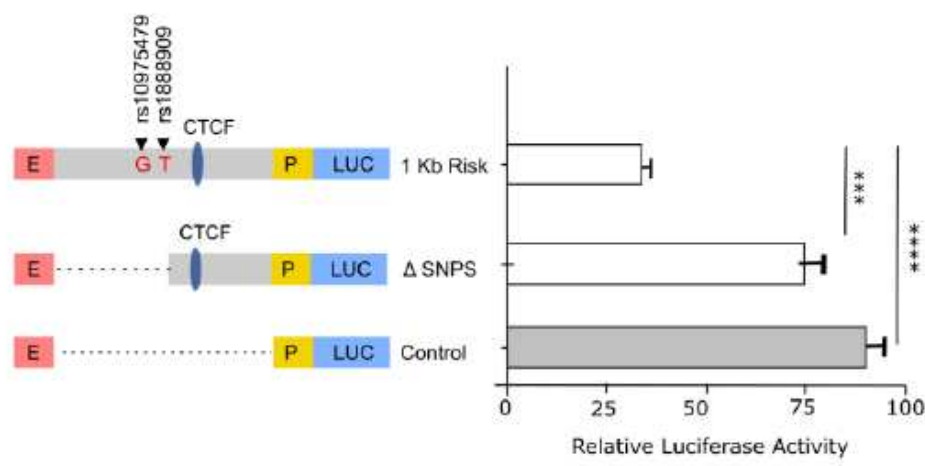
Brain Enhancer Control Somite Promoter GFP

\section{Figure 4}

Impact of the asthma-associated variants in the regulatory property of the $5 \mathrm{~kb}$ region. a In vitro transgenic reporter assay. Luciferase based enhancer barrier assay using $5 \mathrm{~kb}$ constructs (chr9: 6,194,500-6,199,500; hg19) that were cloned between HS2 enhancer (E) and SV40 promoter (P) sequences. SNPs in the construct are noted (black arrowheads). Results are from 3 independent experiments. ${ }^{\star \star \star \star} p<0.0001$, unpaired t-test. b Luciferase activity values of the risk construct is shown as 
fold change over the activity obtained in the non-risk sequence. Results shown represent data from 10 independent experiments. ${ }^{\star \star} \mathrm{p}=0.0014$, two-way ANOVA. $c$ In vivo zebrafish transgenic reporter assay. Green fluorescent protein (GFP) expression 24 hours post fertilization (hpf) in mosaic F0 embryos injected with vectors containing a control sequence (top panel) or $5 \mathrm{~kb}$ interval sequence (bottom panel). $\mathrm{d}$ Comparison between $5 \mathrm{~kb}$ constructs containing risk or non-risk alleles for enhancer blocking property. Data is presented as midbrain/somites EGFP intensity ratio compared with empty gateway vector which has no enhancer blocking activity. ${ }^{\star \star \star *} \mathrm{p}=1.1 \mathrm{e}-5$; $\mathrm{p}=0.041$, ANOVA pairwise T-test. e 1 kb DNA fragment (chr9: 6,197,000-6,197,917; hg19) containing the risk alleles for asthma variants rs10975479 and rs1888909 or a $400 \mathrm{bp}$ deletion in the region harboring those SNPs (chr9: 6,197,399-6,197,914; hg19) were assayed for luciferase reporter activity in K562 cells. Results are representative of 3 independent experiments $* \star * p=0.0005$, unpaired t-test. $f$ Zebrafish reporter assay comparing the $1 \mathrm{~kb} D N A$ fragment and fragment with the $400 \mathrm{bp}$ deletion $(\triangle \mathrm{SNP})$. Data are presented as in (d). 


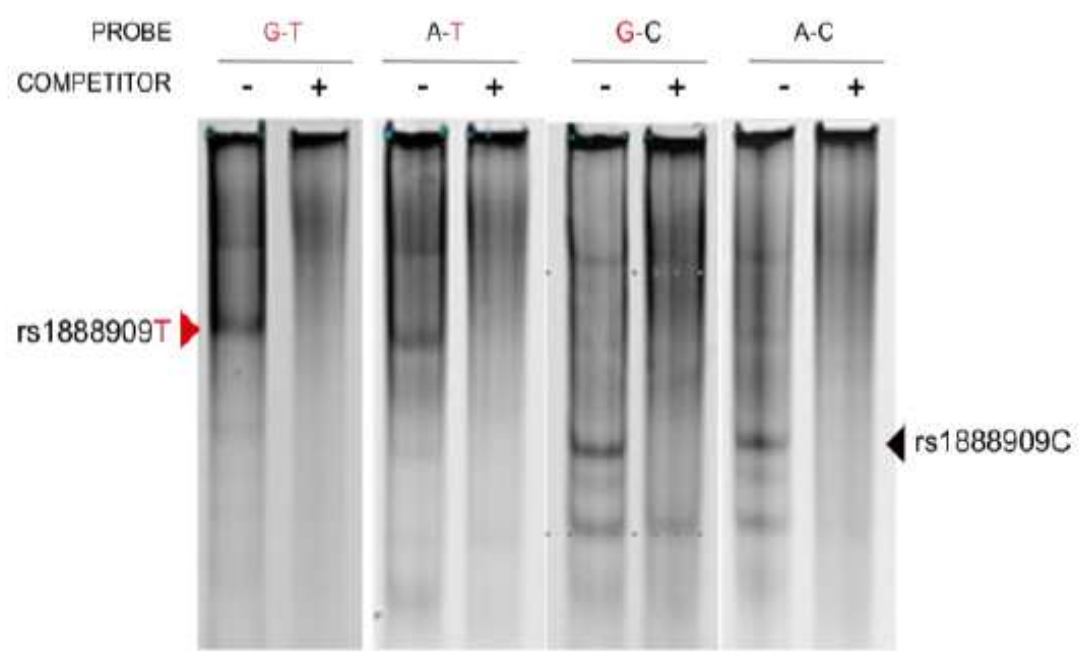

b
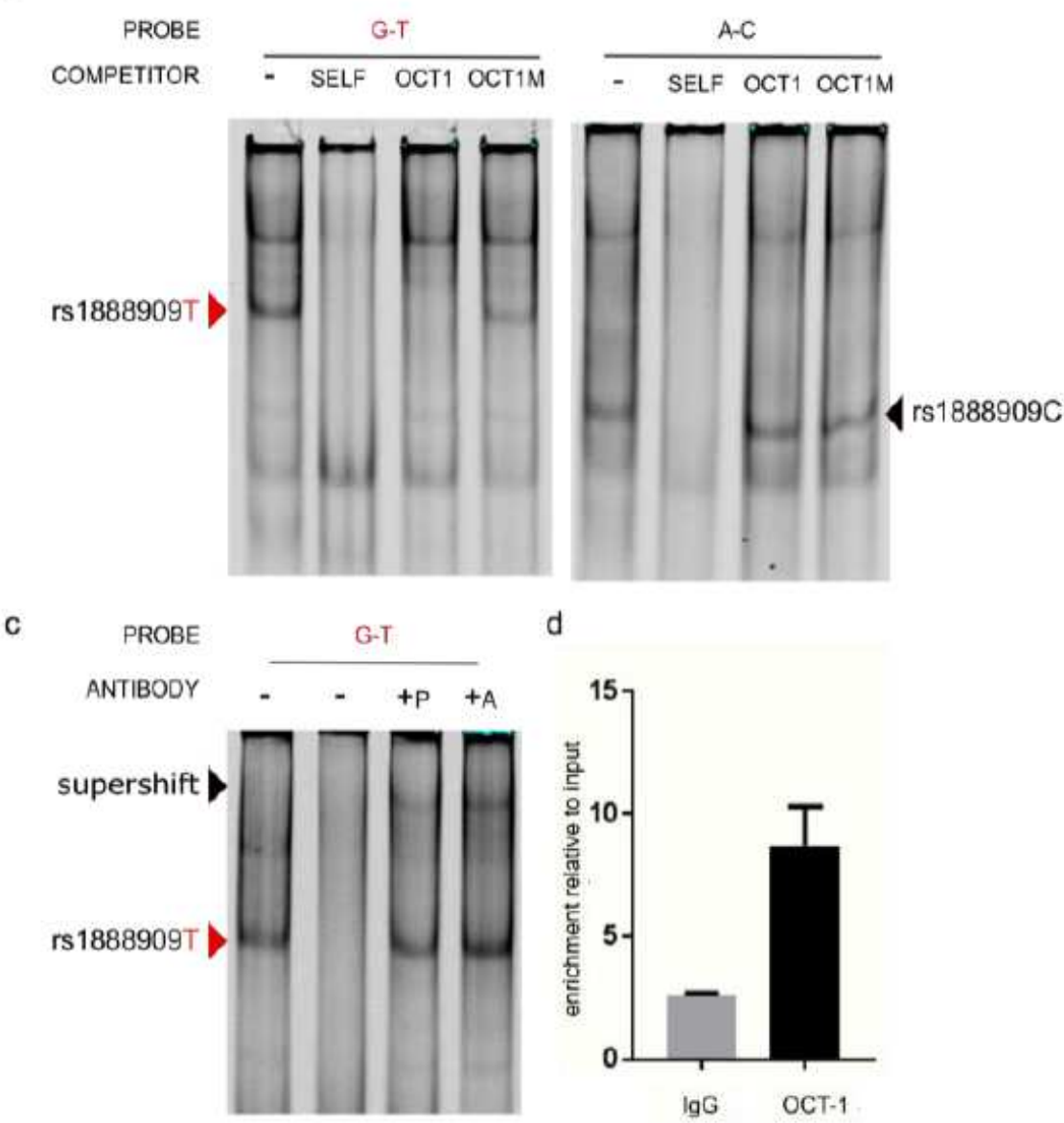

d

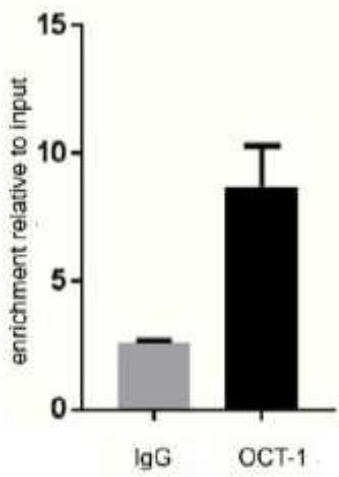

\section{Figure 5}

Regulatory region containing the risk allele rs $1888909 \mathrm{~T}$ selectively binds OCT-1. a Radiolabeled probes carrying the risk (in red) and/or non-risk sequences for SNPs rs10975479 and rs1888909 were incubated with nuclear extract obtained from K562 cells. Different complexes formed by rs 1888909 are marked by red or black arrows. b Cold competition assay with OCT-1 consensus (OCT1) or mutated OCT-1 (OCT1M) oligonucleotides. EMSA probes and oligo competitor (100x molar excess) are noted above each gel. c 
Supershift complex formation with addition of anti-OCT-1 antibody as indicated by the red arrow. $+P$ indicates addition of probe with nuclear extract, followed by incubation with antibody. $+A$ indicates incubation of extract with antibody followed by addition of probe, $+\mathrm{P}$ indicated incubation of extract with probe followed by antibody. d Chromatin immunoprecipitation of H292 chromatin with anti-OCT-1 antibody shows percent enrichment to input chromatin compared to control IgG antibody. Results are representative of 3 independent experiment

a
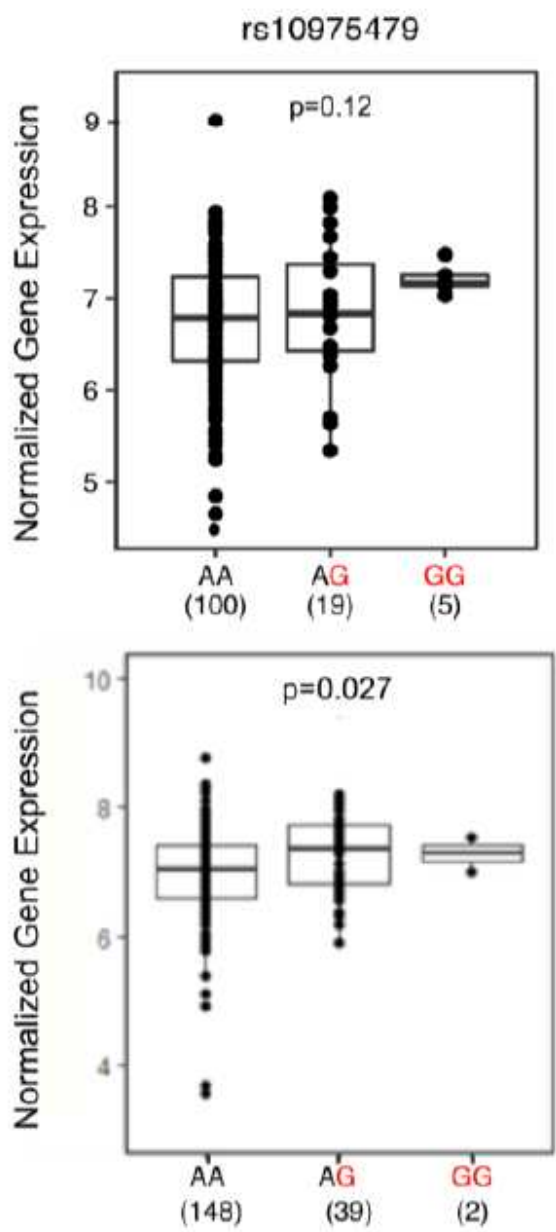

b

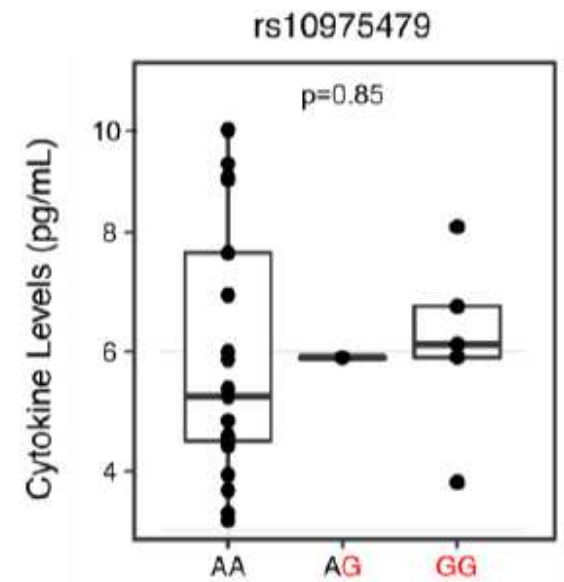

(21)

(1)

(5)

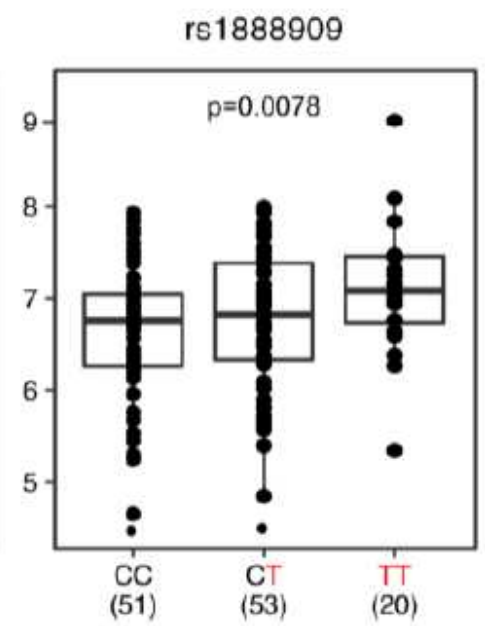

rs1888909

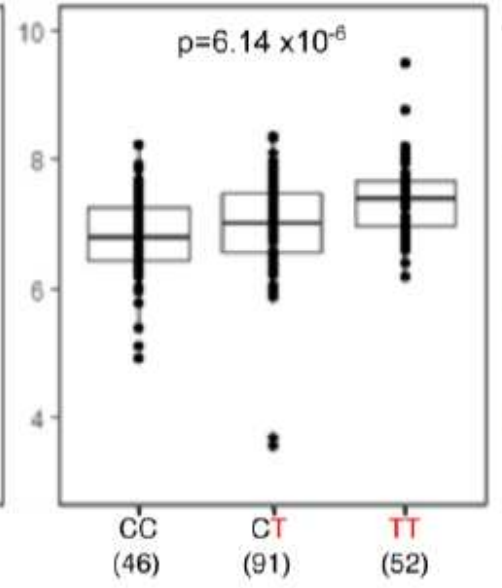

(46)

(91)

rs1888909

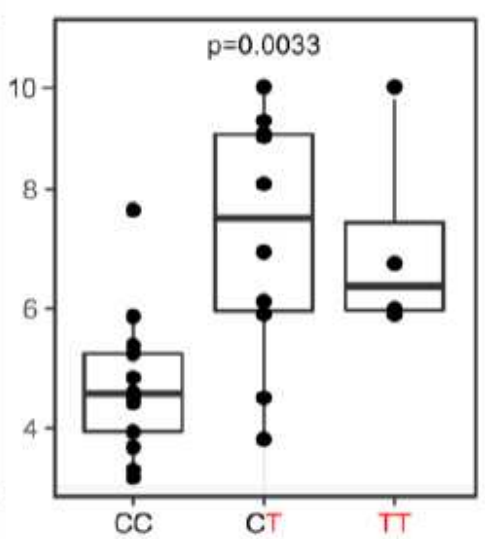

(13)

(10)

(4)
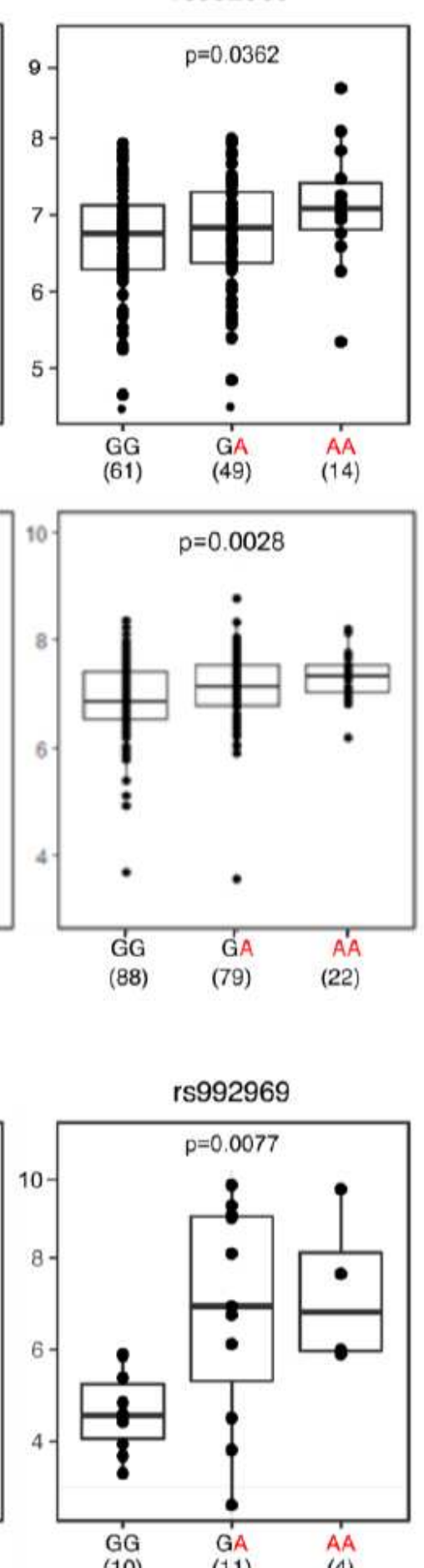

Figure 6 
The rs1888909 (T) and rs992969 (A) alleles are associated with increased IL33 expression and IL-33 protein levels. a Comparison of IL33 expression between genotypes for SNPs rs10975479, rs1888909 and rs992969 from endobronchial brushings from 124 asthmatic and non-asthmatic adult subjects, mostly of European ancestry (upper panels) and nasal epithelial cells from 189 African American children from high risk asthma families (lower panels). b Comparison of IL-33 cytokine levels between genotypes for SNPs rs10975479 ( $n=26)$, rs1888909 $(n=27)$ and rs992969 $(n=25)$ measured in plasma from Hutterite children (all European ancestry). The asthma-associated risk allele at each SNP is highlighted in red ( $x$ axis). The number of subjects per group is shown below the genotype. Boxes indicate the interquartile range, whiskers represent the $95 \%$ confidence intervals. Statistical significance was determined using an additive linear model.

\section{Supplementary Files}

This is a list of supplementary files associated with this preprint. Click to download.

- AneasetalIL33NatCommsupplementaryinformation.pdf 